\title{
A Flocking Algorithm With Individual Agent Destinations and Without a Centralized Leader
}

\author{
Brandon J. Wellman and Jesse B. Hoagg \\ Department of Mechanical Engineering, The University of Kentucky, Lexington, KY \\ 40506-0503
}

\begin{abstract}
We present a multi-agent control method that addresses the combined problem of flocking and destination seeking. The method is completely decentralized, that is, each agent's controller relies on local sensing to determine the relative positions and velocities of nearby agents but does not rely on a centralized flock leader. Each agent has double-integrator dynamics and a potentially unique destination (i.e., position) that the agent must reach. We demonstrate that the flocking-and-destination-seeking control method accomplishes 2 objectives: (i) if an agent is far from its destination, then that agent flocks with nearby agents, and (ii) if an agent is close to its destination, then that agent approaches its destination. The flocking-and-destination-seeking algorithm is demonstrated with several numerical examples.
\end{abstract}

Keywords: Multi-agent systems, flocking, destination seeking

\section{Introduction}

Multi-agent systems have many exciting applications such as distributed sensing, formation flying, cooperative surveillance, and point-to-point mail delivery. For example, autonomous aircraft or spacecraft can fly in formations for distributed sensing $[1,2]$. Coordinated aircraft could be used in a forest-fire scenario to measure wind velocities and thus, predict fire movement. In the agricultural industry, coordinated aircraft could conduct crop surveys. All of these applications require decentralized methods for coordinating and controlling groups of autonomous agents [3].

For coordinated control, each agent relies on sensing to determine the relative positions and velocities of nearby agents. Then, each agent uses these measurements combined with other information such as mission objectives to accomplish tasks, which can include: cohesion, collision avoidance, velocity matching, and guidance. Cohesion attracts an agent to nearby agents, whereas collision avoidance repels an agent from nearby agents (or obstacles). Velocity matching causes nearby agents to approach a consensus velocity, and guidance causes an agent or agents to follow a leader agent or approach a desired destination.

Cohesion and collision avoidance can be addressed using position-formation methods [4-6] or distance-formation methods [7-15]. Position-formation approaches force agents into a configuration using desired relative-position vectors between pairs of agents. In contrast, distance-formation methods induce a configuration using only a desired distance between adjacent agents. In this case, the agents autonomously determine their configuration based 
on the desired interagent distance and initial conditions. A common approach for distance formation is to use potential functions that create attractive forces when nearby agents are too far away and repulsive forces when nearby agents are too close [7-14]. A survey of multi-agent formation methods is presented in [16]. Consensus algorithms [17-20] are used to achieve velocity matching. Approaches that use distance-formation methods for cohesion and collision avoidance, and consensus for velocity matching lead to formations called flocks [10-15].

Agent guidance is often addressed using leader-follower methods [2, 5, 7-13] or destinationseeking methods [6, 21-23]. Leader-follower approaches rely on a centralized leader, who can be an actual or virtual member of the formation and whose real-time position and velocity are known by all agents [8-13] or at least by some [2, 5, 7]. Each agent uses knowledge of the centralized leader and measurements of nearby agents to induce a formation and follow the leader. In contrast, destination-seeking methods (e.g., [6, 21-23]) cause agents to approach desired destinations. The flocking algorithms with leader-follower guidance in [5, 7-13] do not address destination seeking, and the destination-seeking methods in [6, 21-23] do not address flocking. In contrast to [2, 4-16, 21-23], this paper addresses the combined problem of flocking and destination seeking.

The flocking-and-destination-seeking control objective is twofold - if an agent is far from its destination, then it flocks with nearby agents, but ultimately each agent approaches its destination. The flocking-and-destination-seeking algorithm in this paper uses a distanceformation approach for cohesion and collision avoidance, a consensus algorithm for velocity matching, and a destination-seeking method for guidance. The main analytic results in this paper examine the formation properties of agents and provide sufficient conditions for agents to converge to their destinations. Flocking and destination seeking is also considered in [24]; however, the analysis in [24] considers only a single destination and does not examine formation properties.

The flocking-and-destination-seeking algorithm that we present is completely decentralized, namely, each agent's controller does not incorporate a centralized leader and relies on only local sensing to determine the relative positions and velocities of nearby agents. Each agent has knowledge of its own destination but does not require knowledge of other agents' destinations. The controller in this paper achieves multiple objectives, that is, flocking and destination seeking, and thus, extends the work of [2, 4-16, 21-24].

The combined flocking-and-destination-seeking problem has applications such as point-topoint passenger transport and point-to-point mail delivery. For example, consider a group of autonomous ground vehicles on a highway, where each vehicle has a unique destination that it needs to reach. While traveling to the destination, it is beneficial for a vehicle to reduce wind resistance and energy expenditure by drafting off neighboring vehicles. When a vehicle gets close to its destination, it leaves the flock and approaches the destination. The remaining vehicles then form a new flock and repeat the process until all vehicles reach their destinations. Example 2 in Section 7 applies the flocking-and-destination-seeking algorithm to a vehicles-on-a-highway problem. 


\section{Problem Formulation}

Let the positive integer $n$ be the number of agents, and define $\mathcal{I} \triangleq\{1,2, \ldots, n\}$, which is the agent index set. For each $i \in \mathcal{I}$, consider the double-integrator dynamics

$$
\begin{aligned}
\dot{q}_{i}(t) & =p_{i}(t), \\
\dot{p}_{i}(t) & =u_{i}(t),
\end{aligned}
$$

where $t \geq 0 ; q_{i}(0)$ and $p_{i}(0)$ are the initial conditions; and $q_{i}(t) \in \mathbb{R}^{m}, p_{i}(t) \in \mathbb{R}^{m}$, and $u_{i}(t) \in \mathbb{R}^{m}$ are the position, velocity, and control of the $i$ th agent, respectively. Define $\mathcal{P} \triangleq\{(i, j) \in \mathcal{I} \times \mathcal{I}: i \neq j\}$, which is the set of ordered pairs, and let $\|\cdot\|$ denote the Euclidean norm.

The rules for flocking are that agents stay close to one another, avoid collisions, and match velocities [25]. We use these rules to define flocking. Let $T$ be a connected subset of $[0, \infty)$. Then, the agents in $\mathcal{I}$ flock with radius $d>0$ over the interval $T$ if the following conditions hold:

(F1) For all $(i, j) \in \mathcal{P}$ and all $t \in T, q_{i}(t) \neq q_{j}(t)$.

(F2) For all $(i, j) \in \mathcal{P}$ and all $t \in T,\left\|p_{j}(t)-p_{i}(t)\right\| \approx 0$.

(F3) For all $i \in \mathcal{I}$ and all $t \in T, \max _{j \in \mathcal{I} \backslash\{i\}}\left\|q_{j}(t)-q_{i}(t)\right\| \leq d(n-1)$.

(F4) For all $i \in \mathcal{I}$ and all $t \in T, \min _{j \in \mathcal{I} \backslash\{i\}}\left\|q_{j}(t)-q_{i}(t)\right\| \approx d$.

Condition (F1) states that no agents occupy the same position at the same time. Condition (F2) states that all agents have approximately the same velocity. Condition (F3) states that each agent is at most a distance $d(n-1)$ away from its farthest neighbor. Condition (F4) states that each agent maintains a distance of approximately $d$ from its nearest neighbor.

We address not only flocking but also destination seeking. For all $i \in \mathcal{I}$, let $\xi_{i} \in \mathbb{R}^{m}$ be the $i$ th agent's destination. Let $r_{\beta} \geq 0$, and for each $i \in \mathcal{I}$ and each $t \geq 0$, we say the $i$ th agent is far from its destination if $\left\|\xi_{i}-q_{i}(t)\right\|>r_{\beta}$. Assume there exists $t_{\mathrm{f}}>0$ such that for all $t \in\left[0, t_{\mathrm{f}}\right)$, the agents in $\mathcal{I}$ are far from their destinations. In this case, we consider 2 objectives:

(O1) Flocking: The agents in $\mathcal{I}$ flock with radius $d>0$ over a connected subset of $\left[0, t_{\mathrm{f}}\right)$.

(O2) Destination seeking: For all $i \in \mathcal{I}, \lim _{t \rightarrow \infty} q_{i}(t)=\xi_{i}$ and $\lim _{t \rightarrow \infty} p_{i}(t)=0$.

Objective (O1) states that if agents are far from their destinations, then they flock. Objective (O2) states that each agent approaches its destination asymptotically. Unless otherwise stated, all statements in this paper that involve the subscript $i$ are for all $i \in \mathcal{I}$.

\section{Review of Algorithm 1 from [10]}

We review Algorithm 1 from [10], which is a flocking method for agents with doubleintegrator dynamics. Let $\epsilon>0$, and consider $\|\cdot\|_{\epsilon}: \mathbb{R}^{m} \rightarrow[0, \infty)$ defined by

$$
\|x\|_{\epsilon} \triangleq \frac{1}{\epsilon}\left(\underset{3}{\sqrt{1+\epsilon\|x\|^{2}}}-1\right) .
$$


Note that $\|\cdot\|_{\epsilon}$ is continuously differentiable on $\mathbb{R}^{m}$, but $\|\cdot\|_{\epsilon}$ is not a norm on $\mathbb{R}^{m}$. Define $\sigma_{\epsilon}: \mathbb{R}^{m} \rightarrow \mathbb{R}^{m}$ by

$$
\sigma_{\epsilon}(x) \triangleq\left(\frac{\partial}{\partial x}\left[\|x\|_{\epsilon}\right]\right)^{\mathrm{T}}=\frac{x}{1+\epsilon\|x\|_{\epsilon}} .
$$

Next, let $h \in(0,1)$, and define $\rho_{h}:[0, \infty) \rightarrow[0,1]$ by

$$
\rho_{h}(\eta) \triangleq \begin{cases}1, & \text { if } \eta \in[0, h), \\ \frac{1}{2}+\frac{1}{2} \cos \pi \frac{\eta-h}{1-h}, & \text { if } \eta \in[h, 1] \\ 0, & \text { if } \eta \in(1, \infty),\end{cases}
$$

which decreases from 1 to 0 as $\eta$ increases from 0 to $\infty$, and the rate of change of $\rho_{h}$ depends on $h$. Let $b \geq a>0$, define $c \triangleq(b-a) / \sqrt{4 a b}$, and consider $\phi: \mathbb{R} \rightarrow(-b, a)$ defined by

$$
\phi(\eta) \triangleq \frac{1}{2}\left[\frac{(a+b)(\eta+c)}{\sqrt{1+(\eta+c)^{2}}}+(a-b)\right]
$$

which is a sigmoidal function.

Next, let $r_{\mathrm{c}}>0$ be the communication radius, which is the maximum distance at which an agent can sense another agent's relative position and relative velocity. For all $t \geq 0$, define the neighbor set $\mathcal{N}_{i}(t) \triangleq\left\{j \in \mathcal{I} \backslash\{i\}:\left\|q_{j}(t)-q_{i}(t)\right\|<r_{\mathrm{c}}\right\}$, which is the set of agents whose distance to the $i$ th agent is no greater than the communication radius $r_{\mathrm{c}}$ at time $t$. Let $d \in\left(0, r_{\mathrm{c}}\right]$ be the flock radius, which is the desired distance between agents in the flock.

For all $t \geq 0$, define $q(t) \triangleq\left[\begin{array}{lll}q_{1}^{\mathrm{T}}(t) & \cdots & q_{n}^{\mathrm{T}}(t)\end{array}\right]^{\mathrm{T}}$ and $p(t) \triangleq\left[\begin{array}{lll}p_{1}^{\mathrm{T}}(t) & \cdots & p_{n}^{\mathrm{T}}(t)\end{array}\right]^{\mathrm{T}}$. Then, [10, Algorithm 1] considers the control $u_{i}(t)=v_{i}(q(t), p(t))$, where

$$
v_{i}(q, p) \triangleq \underbrace{\sum_{j \in \mathcal{N}_{i}} \rho_{h}\left(\frac{\left\|q_{j}-q_{i}\right\|_{\epsilon}}{\left\|r_{\mathrm{c}}\right\|_{\epsilon}}\right) \Phi\left(q_{j}-q_{i}\right)}_{\text {Flock attraction and repulsion }}+\underbrace{\sum_{j \in \mathcal{N}_{i}} \rho_{h}\left(\frac{\left\|q_{j}-q_{i}\right\|_{\epsilon}}{\left\|r_{\mathrm{c}}\right\|_{\epsilon}}\right)\left[p_{j}-p_{i}\right]}_{\text {Velocity consensus }},
$$

and $\Phi: \mathbb{R}^{m} \rightarrow \mathbb{R}^{m}$ is defined by $\Phi(x) \triangleq \phi\left(\|x\|_{\epsilon}-\|d\|_{\epsilon}\right) \sigma_{\epsilon}(x)$.

For each $t \geq 0$ and each $j \in \mathcal{N}_{i}(t)$, the flock-attraction-and-repulsion term in (7) is such that the $i$ th agent is attracted to the $j$ th agent if $\left\|q_{j}(t)-q_{i}(t)\right\|>d$, and repelled from the $j$ th agent if $\left\|q_{j}(t)-q_{i}(t)\right\|<d$. The velocity-consensus term in (7) attempts to match the $i$ th agent's velocity with a weighted average of the velocities of all agents in the neighbor set.

The parameters in the control (7) are $\epsilon, h, a$, and $b$. Increasing $\epsilon$ decreases the strength of the flock-attraction-and-repulsion term relative to the strength of the velocity-consensus term. Increasing $h$ increases the rate of change of $\rho_{h}$. Increasing $a$ increases the strength of attraction relative to the strength of repulsion and velocity consensus. Increasing $b$ increases the strength of repulsion relative to the strength of attraction and velocity consensus.

Theorem 1 of [10] provides conditions such that the agents with dynamics (1), (2) and control $u_{i}=v_{i}$ form at least one flock. However, the control $u_{i}=v_{i}$ can cause fragmentation, that is, $u_{i}=v_{i}$ can result in multiple flocks rather than a single flock. Thus, the control $u_{i}=v_{i}$ may violate (F3). In contrast, [10, Algorithm 2] is shown to force the agents in $\mathcal{I}$ to flock without fragmentation. However, [10, Algorithm 2] relies on a centralized flock leader whose position and velocity are known by each agent and used in each agent's control. Neither [10, Algorithm 1] nor [10, Algorithm 2] address destination seeking and thus cannot 
be used to achieve (O2).

\section{Flocking and Destination Seeking}

We present a control that combines flock-attraction-and-repulsion and velocity-consensus terms with additional terms to achieve (O1) and (O2). For all $t \geq 0$, define the attraction set $\mathcal{A}_{i}(t) \triangleq\left\{j \in \mathcal{I} \backslash\{i\}: d \leq\left\|q_{j}(t)-q_{i}(t)\right\|<r_{\mathrm{c}}\right\} \subseteq \mathcal{N}_{i}(t)$, which is the set of agents whose distances from the $i$ th agent are between the flock and communication radii. Define

$$
A_{i}(q) \triangleq \sum_{j \in \mathcal{A}_{i}} \rho_{h}\left(\frac{\left\|q_{j}-q_{i}\right\|_{\epsilon}}{\left\|r_{\mathrm{c}}\right\|_{\epsilon}}\right) \Phi\left(q_{j}-q_{i}\right)
$$

which has the same form as the flock-attraction-and-repulsion term in (7) except that the neighbor set $\mathcal{N}_{i}$ is replaced by the attraction set $\mathcal{A}_{i}$. Next, for all $t \geq 0$, define the repulsion set $\mathcal{R}_{i}(t) \triangleq\left\{j \in \mathcal{I} \backslash\{i\}:\left\|q_{j}(t)-q_{i}(t)\right\|<d\right\} \subseteq \mathcal{N}_{i}(t)$, which is the set of agents whose distances to the $i$ th agent are less than the flock radius. Define

$$
R_{i}(q) \triangleq \sum_{j \in \mathcal{R}_{i}} \rho_{h}\left(\frac{\left\|q_{j}-q_{i}\right\|_{\epsilon}}{\left\|r_{c}\right\|_{\epsilon}}\right) \Phi\left(q_{j}-q_{i}\right)
$$

which has the same form as the flock-attraction-and-repulsion term in (7) except that the neighbor set $\mathcal{N}_{i}$ is replaced by the repulsion set $\mathcal{R}_{i}$. For all $t \geq 0, \mathcal{A}_{i}(t) \cup \mathcal{R}_{i}(t)=\mathcal{N}_{i}(t)$, and $A_{i}(q)+R_{i}(q)$ is equal to the flock-attraction-and-repulsion term in (7). Next, define

$$
C_{i}(q, p) \triangleq \sum_{j \in \mathcal{N}_{i}} \rho_{h}\left(\frac{\left\|q_{j}-q_{i}\right\|_{\epsilon}}{\left\|r_{\mathrm{c}}\right\|_{\epsilon}}\right)\left[p_{j}-p_{i}\right]
$$

which is the velocity-consensus term in (7). Therefore, $v_{i}(q, p)=A_{i}(q)+R_{i}(q)+C_{i}(q, p)$.

Let $r_{\alpha} \in\left[0, r_{\beta}\right]$, and for all $t \geq 0$, we say that the $i$ th agent is close to its destination if $\left\|\xi_{i}-q_{i}(t)\right\| \leq r_{\alpha}$. Let $\mu:[0, \infty) \rightarrow[0,1]$ be a continuous function that satisfies the following properties:

(M1) If $\eta \geq r_{\beta}$, then $\mu(\eta)=1$.

(M2) If $\eta<r_{\alpha}$, then $\mu(\eta)=0$.

(M3) $\partial \mu(\|x\|) / \partial x$ is continuous on $\mathbb{R}^{m}$.

If $r_{\beta}>r_{\alpha} \geq 0$, then an example $\mu$ that satisfies (M1)-(M3) is

$$
\mu(\eta)= \begin{cases}0, & \text { if } \eta \leq r_{\alpha}, \\ \frac{1}{2}+\frac{1}{2} \cos \pi \frac{r_{\beta}^{2}-\eta^{2}}{r_{\beta}^{2}-r_{\alpha}^{2}}, & \text { if } r_{\alpha}<\eta<r_{\beta}, \\ 1, & \text { if } \eta \geq r_{\beta} .\end{cases}
$$

Note that if $r_{\alpha}=r_{\beta}=0$, then (M1) implies that $\mu=1$.

For all $t \geq 0$, define $z_{i}(t) \triangleq \xi_{i}-q_{i}(t)$, which is the vector from the $i$ th agent to its destination. If the $i$ th agent is far from its destination, then the $i$ th agent's objective is to flock with nearby agents. In contrast, if the $i$ th agent is close to its destination, then the $i$ th agent's objective is not to flock but rather to reach its destination. 
The following result considers a control that consists of the flock attraction $\mu\left(\left\|z_{i}\right\|\right) A_{i}(q)$, flock repulsion $R_{i}(q)$, and velocity consensus $\mu\left(\left\|z_{i}\right\|\right) C_{i}(q, p)$. The result is confirmed by direct calculation.

Proposition 1. Consider (1) and (2), where for all $t \geq 0$,

$$
u_{i}\left(q(t), p(t), z_{i}(t)\right)=\mu\left(\left\|z_{i}(t)\right\|\right) A_{i}(q(t))+R_{i}(q(t))+\mu\left(\left\|z_{i}(t)\right\|\right) C_{i}(q(t), p(t)) .
$$

Then, the following statements hold:

(a) If $\left\|z_{i}(t)\right\| \geq r_{\beta}$, then $u_{i}\left(q(t), p(t), z_{i}(t)\right)=v_{i}(q(t), p(t))$.

(b) If $\left\|z_{i}(t)\right\|<r_{\alpha}$, then $u_{i}\left(q(t), p(t), z_{i}(t)\right)=R_{i}(q(t))$.

(c) $u_{i}\left(q, p, z_{i}\right)$ is continuously differentiable on $\mathbb{R}^{n m} \times \mathbb{R}^{n m} \times \mathbb{R}^{m}$.

Part (a) states that if the $i$ th agent is far from its destination, then (12) is equivalent to (7). Part (b) states that if the $i$ th agent is close to its destination, then (12) consists of only flock repulsion. Thus, if $\left\|z_{i}(t)\right\|<r_{\alpha}$, then the $i$ th agent is neither attracted to nor matches velocities with nearby agents.

We now describe two additional desired properties for the agents in $\mathcal{I}$. Let $r_{2}>r_{1}>0$. If $\left\|z_{i}(t)\right\|>r_{2}$, then we want the $i$ th agent's speed $\left\|p_{i}(t)\right\|$ to tend to the flocking speed $p_{\mathrm{f}}>0$. In contrast, if $\left\|z_{i}(t)\right\| \leq r_{1}$, then we want the $i$ th agent to approach its destination. Specifically, if $\left\|z_{i}(t)\right\| \leq r_{1}$, then we want $q_{i}$ to satisfy the differential equation

$$
\ddot{y}_{i}(t)+2 \zeta \omega_{\mathrm{n}} \dot{y}_{i}(t)+\omega_{\mathrm{n}}^{2} y_{i}(t)=\omega_{\mathrm{n}}^{2} \xi_{i},
$$

where $y_{i}(t) \in \mathbb{R}^{m}, \omega_{\mathrm{n}}>0$, and $\zeta>0$.

To achieve these properties, we consider a control that includes destination-seeking and damping terms. Let $\gamma \in\left(0, \omega_{\mathrm{n}}^{2} r_{1}\right)$, and consider $\nu:[0, \infty) \rightarrow\left(0, \omega_{\mathrm{n}}^{2}\right]$ defined by

$$
\nu(\eta) \triangleq \begin{cases}\omega_{\mathrm{n}}^{2}, & \text { if } \eta \leq r_{1}, \\ \frac{1}{2}\left(\omega_{\mathrm{n}}^{2}+\frac{\gamma}{\eta}\right)-\frac{1}{2}\left(\omega_{\mathrm{n}}^{2}-\frac{\gamma}{\eta}\right) \cos \pi \frac{r_{2}^{2}-\eta^{2}}{r_{2}^{2}-r_{1}^{2},} & \text { if } r_{1}<\eta<r_{2}, \\ \frac{\gamma}{\eta}, & \text { if } \eta \geq r_{2},\end{cases}
$$

which is continuously differentiable on $[0, \infty)$ and shown in Figure 1 . We use the destinationseeking control $\nu\left(\| z_{i}||\right) z_{i}$ to attract the $i$ th agent to its destination.

Next, consider $\kappa:[0, \infty) \rightarrow(-\infty, 0)$ defined by

$$
\kappa(\eta) \triangleq \begin{cases}-2 \zeta \omega_{\mathrm{n}}, & \text { if } \eta \leq r_{1}, \\ -\frac{1}{2}\left(2 \zeta \omega_{\mathrm{n}}+\frac{\gamma}{p_{\mathrm{f}}}\right)-\frac{1}{2}\left(2 \zeta \omega_{\mathrm{n}}-\frac{\gamma}{p_{\mathrm{f}}}\right) \cos \pi \frac{r_{2}^{2}-\eta^{2}}{r_{2}^{2}-r_{1}^{2}}, & \text { if } r_{1}<\eta<r_{2}, \\ -\frac{\gamma}{p_{\mathrm{f}}}, & \text { if } \eta \geq r_{2},\end{cases}
$$

which is continuously differentiable on $[0, \infty)$ and shown in Figure 2. We use the damping control $\kappa\left(\| z_{i}||\right) p_{i}$ to oppose the velocity $p_{i}$. 


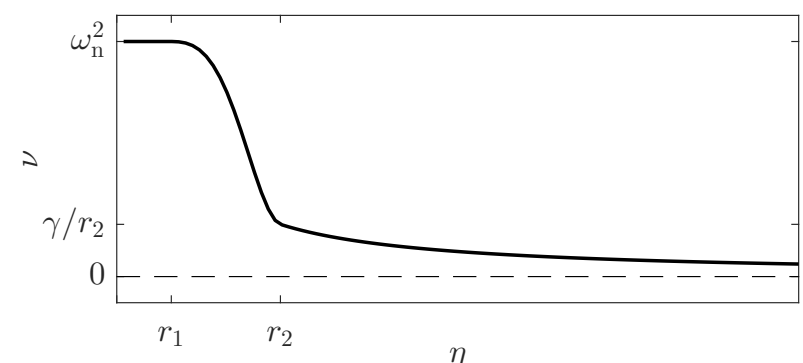

Figure 1: A plot of $\nu:[0, \infty) \rightarrow\left(0, \omega_{\mathrm{n}}^{2}\right]$, which is continously differentiable on $[0, \infty)$. For all $\eta \leq r_{1}$, $\nu(\eta)=\omega_{\mathrm{n}}^{2}$, and for all $\eta \geq r_{2}, \nu(\eta)=\gamma / \eta$.

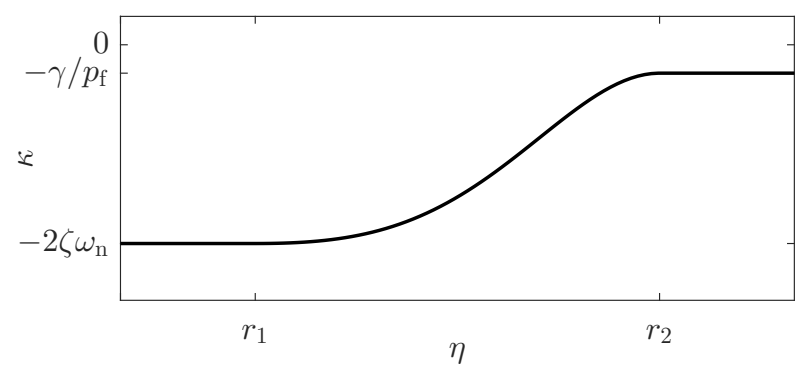

Figure 2: A plot of $\kappa:[0, \infty) \rightarrow(-\infty, 0)$, which is continuously differentiable on $[0, \infty)$. For all $\eta \leq r_{1}$, $\kappa(\eta)=-2 \zeta \omega_{\mathrm{n}}$, and for all $\eta \geq r_{2}, \kappa(\eta)=-\gamma / p_{\mathrm{f}}$.

Define $\xi \triangleq\left[\begin{array}{lll}\xi_{1}^{\mathrm{T}} & \cdots & \xi_{n}^{\mathrm{T}}\end{array}\right]^{\mathrm{T}}$. The following result demonstrates that a control composed of destination seeking $\nu\left(\left\|z_{i}\right\|\right) z_{i}$ and damping $\kappa\left(\left\|z_{i}\right\|\right) p_{i}$ forces the $i$ th agent's position $q_{i}$ to converge asymptotically to its destination $\xi_{i}$.

Proposition 2. Consider (1) and (2), where for all $t \geq 0$,

$$
u_{i}\left(z_{i}(t), p_{i}(t)\right)=\nu\left(\left\|z_{i}(t)\right\|\right) z_{i}(t)+\kappa\left(\left\|z_{i}(t)\right\|\right) p_{i}(t) .
$$

Then, the following statements hold:

(a) $(q(t), p(t)) \equiv(\xi, 0)$ is a globally asymptotically stable equilibrium of (1), (2), and (16).

(b) There exists $t_{1}>0$ such that, for all $t \geq t_{1},\left\|z_{i}(t)\right\| \leq r_{1}$ and $q_{i}(t)$ satisfies $\ddot{q}_{i}(t)+$ $2 \zeta \omega_{\mathrm{n}} \dot{q}_{i}(t)+\omega_{\mathrm{n}}^{2} q_{i}(t)=\omega_{\mathrm{n}}^{2} \xi_{i}$.

(c) Assume $\left\|z_{i}(0)\right\|>r_{2}$. Then, there exists $t_{0}>0$ such that, for all $t \in\left[0, t_{0}\right)$, $u_{i}\left(z_{i}(t), p_{i}(t)\right)=\gamma z_{i}(t) /\left\|z_{i}(t)\right\|-\gamma p_{i}(t) / p_{\mathrm{f}}$.

(d) $u_{i}\left(z_{i}, p_{i}\right)$ is continuously differentiable on $\mathbb{R}^{m} \times \mathbb{R}^{m}$.

Part (b) states that there exists a time after which an agent approaches its destination with the dynamics (13) of a damped oscillator. If an agent's initial position is greater than $r_{2}$ from its destination, then part (c) provides an expression for the destination-seeking-and-damping control.

If $\left\|z_{i}(t)\right\|>r_{2}$, then (14) and (15) imply that (16) is equivalent to $u_{i}\left(z_{i}(t), p_{i}(t)\right)=$ $\gamma z_{i}(t) /\left\|z_{i}(t)\right\|-\gamma p_{i}(t) / p_{\mathrm{f}}$. In this case, it follows from $(2)$ that $p_{i}(t)$ satisfies

$$
\dot{p}_{i}(t)=-\gamma p_{i}(t) / p_{\mathrm{f}}+\gamma z_{i}(t) /\left\|z_{i}(t)\right\|,
$$

which can be viewed as a linear time-invariant differential equation with the bounded input $\gamma z_{i}(t) /\left\|z_{i}(t)\right\|$. If the destination $\xi_{i}$ is infinitely far from the position $q_{i}$ (i.e., $\left\|z_{i}\right\|=\infty$ ), then $z_{i} /\left\|z_{i}\right\|$ is a constant unit vector. In this case, (17) implies that $p_{i}(t)$ tends toward $p_{\mathrm{f}} z_{i}(t) /\left\|z_{i}(t)\right\|$, and the speed $\left\|p_{i}(t)\right\|$ tends toward $p_{\mathrm{f}}$. Thus, if $\left\|z_{i}(t)\right\|$ is sufficiently large, then the $i$ th agent has forces that drive its speed $\left\|p_{i}(t)\right\|$ toward $p_{\mathrm{f}}$. 
We now combine the flock-attraction, flock-repulsion, velocity-consensus, destinationseeking, and damping terms to form the control

$$
u_{i}\left(q, p, z_{i}\right)=\underbrace{\mu\left(\left\|z_{i}\right\|\right) A_{i}(q)}_{\text {Flock attraction }}+\underbrace{R_{i}(q)}_{\begin{array}{c}
\text { Flock } \\
\text { repulsion }
\end{array}}+\underbrace{\mu\left(\left\|z_{i}\right\|\right) C_{i}(q, p)}_{\begin{array}{c}
\text { Velocity } \\
\text { consensus }
\end{array}}+\underbrace{\nu\left(\left\|z_{i}\right\|\right) z_{i}}_{\begin{array}{c}
\text { Destination } \\
\text { seeking }
\end{array}}+\underbrace{\kappa\left(\left\|z_{i}\right\|\right) p_{i}}_{\text {Damping }} .
$$

Since (18) is the sum of the controllers in Propositions 1 and 2, it follows that (18) is continuously differentiable on $\mathbb{R}^{n m} \times \mathbb{R}^{n m} \times \mathbb{R}^{m}$. In addition, we note that flock attraction, flock repulsion, and destination seeking are bounded.

The parameters of $u_{i}$ are $\epsilon, h, a, b, r_{\alpha}, r_{\beta}, \mu, r_{1}, r_{2}, \gamma, p_{\mathrm{f}}, \zeta$, and $\omega_{\mathrm{n}}$, where the first 6 parameters and the function $\mu$ apply to the flock-attraction, flock-repulsion, and velocityconsensus terms, and the latter 6 parameters apply to the destination-seeking and damping terms. Note that $\epsilon, h, a$, and $b$ are discussed in Section 3. We now discuss $r_{\alpha}, r_{\beta}, \mu, r_{1}, r_{2}, \gamma$, $p_{\mathrm{f}}, \zeta$, and $\omega_{\mathrm{n}}$.

The radii $r_{\alpha}, r_{\beta}, r_{1}$, and $r_{2}$ determine the $i$ th agent's control behavior for specific distances from its destination $\xi_{i}$. If $\left\|z_{i}(t)\right\|>r_{\beta}$, then the $i$ th agent and nearby agents attempt to flock. If $\left\|z_{i}(t)\right\| \leq r_{\alpha}$, then the $i$ th agent is repelled from nearby agents but does not attempt to flock. If $\left\|z_{i}(t)\right\|>r_{2}$, then the $i$ th agent has forces that drive its speed $\left\|p_{i}(t)\right\|$ toward $p_{\mathrm{f}}$. If $\left\|z_{i}(t)\right\| \leq r_{1}$, then the $i$ th agent has forces that make it behave like the damped oscillator. While $r_{1}=r_{\alpha}$ and $r_{2}=r_{\beta}$ is an intuitive choice, this is not required.

The function $\mu$ determines the strength of flock attraction and velocity consensus relative to flock repulsion, destination seeking, and damping. If $\mu\left(\left\|z_{i}(t)\right\|\right)=1$, then the combination of flock-attraction, flock-repulsion, and velocity-consensus terms in (18) is equal to the combination of flock-attraction-and-repulsion and velocity-consensus terms in (7). As $\mu\left(\left\|z_{i}(t)\right\|\right)$ decreases, the strength of flock attraction and velocity consensus decreases relative to flock repulsion, destination seeking, and damping. If $\mu\left(\left\|z_{i}(t)\right\|\right)=0$, then the flock-attraction and velocity-consensus terms are zero.

For $\left\|z_{i}(t)\right\| \geq r_{2}, \gamma$ is the magnitude of the destination-seeking term $\nu\left(\left\|z_{i}(t)\right\|\right) z_{i}(t)$. In this case, increasing $\gamma$ increases the $i$ th agent's attraction to its destination. For $\left\|z_{i}(t)\right\| \geq r_{2}$, increasing $\gamma$ also increases the rate at which the $i$ th agent's speed $\left\|p_{i}(t)\right\|$ tends to $p_{\mathrm{f}}$. The flocking speed $p_{\mathrm{f}}$, which is the desired speed of agents, can be chosen for a variety of reasons such as fuel or time constraints.

Finally, $\zeta$ and $\omega_{\mathrm{n}}$ are the damping ratio and natural frequency of the dynamics (13) that govern the desired behavior of an agent when it's near its destination.

\section{Flocking Analysis}

In this section, we analyze the flocking behavior of agents with control (18). Since (18) is designed to drive each agent to its destination, the agents flock over at most a finite time interval before approaching their destinations. To analyze flocking, we impose assumptions on the destinations so that we can use asymptotic analysis to examine flocking. In particular, we impose assumptions that preclude agents from reaching their destinations, and thus allow us to demonstrate flocking with asymptotic analysis.

To analyze asymptotic flocking, we could consider destinations $\xi_{1}, \ldots, \xi_{n}$ that are infinitely 
far from the initial positions $q_{1}(0), \ldots, q_{n}(0)$. However, in this case, $z_{i}=\xi_{i}-q_{i}$ is not well defined. Instead, we impose the following assumptions regarding the agents' destinations:

(A1) All agents have the same destination, and this destination moves with constant velocity $\chi \in \mathbb{R}^{m}$, where $\|\chi\|=p_{\mathrm{f}}$.

(A2) For all $t \geq 0,\left\|z_{i}(t)\right\|>\max \left\{r_{\beta}, r_{2}\right\}$.

These assumptions are used for flocking analysis only and apply only to this section of the paper. We refer to (A1) as the moving-destination assumption. Assumption (A2) combined with (M1), (14), and (15) implies that $\mu\left(\left\|z_{i}(t)\right\|\right)=1, \nu\left(\left\|z_{i}(t)\right\|\right) z_{i}(t)=\gamma z_{i}(t) /\left\|z_{i}(t)\right\|$, and $\kappa\left(\left\|z_{i}(t)\right\|\right)=-\gamma / p_{\mathrm{f}}$. Thus, (A2) implies that the control (18) depends on the direction $z_{i}(t) /\left\|z_{i}(t)\right\|$ but not the distance $\left\|z_{i}(t)\right\|$. Therefore, the control (18) with the movingdestination assumption (A1) is equivalent to the control (18) with a non-moving destination that is infinitely far from the initial positions. Thus, (A1) and (A2) are in some sense equivalent to an infinitely far non-moving destination. Asymptotic flocking under (A1) and (A2) suggests that if the initial positions $q_{1}(0), \ldots, q_{n}(0)$ are sufficiently far from the destinations $\xi_{1}, \ldots, \xi_{n}$, and the destinations are sufficiently close together relative to the distances $\left\|z_{1}\right\|, \ldots,\left\|z_{n}\right\|$, then the agents flock over a finite time interval.

It follows from (A1) that the destination dynamics are $\dot{\xi}_{i}(t)=\chi$, where $\xi_{i}(t) \in \mathbb{R}^{m}$ is the $i$ th agent's destination, $\xi_{i}(0) \in \mathbb{R}^{m}$ is the initial position, and $\xi_{1}(0)=\cdots=\xi_{n}(0)$. For all $t \geq 0$, define $w_{i}(t) \triangleq \dot{z}_{i}(t)=\chi-p_{i}(t)$. Thus, (1) and (2) imply that, for all $t \geq 0$,

$$
\begin{aligned}
\dot{z}_{i}(t) & =w_{i}(t), \\
\dot{w}_{i}(t) & =-u_{i}(t) .
\end{aligned}
$$

For all $t \geq 0$, define $z(t) \triangleq\left[\begin{array}{lll}z_{1}^{\mathrm{T}}(t) & \cdots & z_{n}^{\mathrm{T}}(t)\end{array}\right]^{\mathrm{T}}$ and $w(t) \triangleq\left[\begin{array}{lll}w_{1}^{\mathrm{T}}(t) & \cdots & w_{n}^{\mathrm{T}}(t)\end{array}\right]^{\mathrm{T}}$.

We use Lyapunov-like analysis to examine the asymptotic properties of (1), (2), and (18) under (A1) and (A2). Consider $\psi:[0, \infty) \rightarrow[0, \infty)$ defined by

$$
\psi(\eta) \triangleq \int_{\|d\|_{\epsilon}}^{\eta} \rho_{h}\left(\frac{s}{\left\|r_{\mathrm{c}}\right\|_{\epsilon}}\right) \phi\left(s-\|d\|_{\epsilon}\right) \mathrm{d} s
$$

If $\eta=\|d\|_{\epsilon}$, then $\psi(\eta)=0$; otherwise, $\psi(\eta)>0$. Furthermore, consider $\psi_{\mathrm{s}}: \mathbb{R}^{n m} \rightarrow[0, \infty)$ defined by

$$
\psi_{\mathrm{s}}(q) \triangleq \frac{1}{2} \sum_{(i, j) \in \mathcal{P}} \psi\left(\left\|q_{j}-q_{i}\right\|_{\epsilon}\right) .
$$

If, for all $(i, j) \in \mathcal{P},\left\|q_{j}-q_{i}\right\|=d$, then $\psi_{\mathrm{s}}(q)=0$; otherwise, $\psi_{\mathrm{s}}(q)>0$. Consider the Lyapunov-like function $W: \mathbb{R}^{n m} \times \mathbb{R}^{n m} \rightarrow[0, \infty)$ defined by

$$
W(z, w) \triangleq \psi_{\mathrm{s}}(z)+\sum_{i \in \mathcal{I}} \gamma\left\|z_{i}\right\|-\frac{\gamma}{p_{\mathrm{f}}} \chi^{\mathrm{T}} z_{i}+\frac{1}{2}\|w\|^{2} .
$$

Since $\|\chi\|=p_{\mathrm{f}}$, it follows that $\sum_{i \in \mathcal{I}} \gamma\left\|z_{i}\right\|-\frac{\gamma}{p_{\mathrm{f}}} \chi^{\mathrm{T}} z_{i} \geq \sum_{i \in \mathcal{I}} \gamma\left\|z_{i}\right\|-\frac{\gamma}{p_{\mathrm{f}}}\|\chi\|\left\|z_{i}\right\|=0$. Since, in addition, $\psi_{\mathrm{s}} \geq 0$, it follows that, for all $(z, w) \in \mathbb{R}^{n m} \times \mathbb{R}^{n m}, W(z, w) \geq 0$. If, for all $(i, j) \in \mathcal{P}$, $\left\|z_{j}-z_{i}\right\|=d, \chi^{\mathrm{T}} z_{i}=p_{\mathrm{f}}\left\|z_{i}\right\|$, and $w_{i}=0$, then $W(z, w)=0$; otherwise, $W(z, w)>0$.

The following result provides sufficient conditions such that the agents do not collide; all agents match velocity; the maximum distance between any 2 agents is asymptotically 
bounded by $d(n-1)$; and the derivative of $\psi_{\mathrm{s}}$ along the trajectories of $(18)-(20)$ tends to 0 . The proof is in Appendix B.

Theorem 1. Consider the closed-loop system (18)-(20), where the initial conditions are $z_{i}(0) \in \mathbb{R}^{m}$ and $w_{i}(0) \in \mathbb{R}^{m}$. Assume (A1) and (A2) hold. Then, the following statements hold:

(a) If $W(z(0), w(0))<\psi(0)$, then, for all $(i, j) \in \mathcal{P}$ and all $t \geq 0, q_{j}(t) \neq q_{i}(t)$.

(b) $\lim _{t \rightarrow \infty} p_{i}(t)=\chi$.

(c) Assume $r_{\mathrm{c}}$ is such that, for all $(i, j) \in \mathcal{P}$ and all $t \geq 0,\left\|r_{\mathrm{c}}\right\|\left\|_{\epsilon}>\right\| q_{j}(t)-q_{i}(t)\|\|_{\epsilon} / h$. Then, for all $\delta>0$, there exists $t_{1} \geq 0$ such that, for all $t \geq t_{1}, \max _{(i, j) \in \mathcal{P}}\left\|q_{j}(t)-q_{i}(t)\right\| \leq$ $d(n-1)+\delta$.

(d) $\lim _{t \rightarrow \infty} \partial \psi_{\mathrm{s}}(z) /\left.\partial z\right|_{z=z(t)}=0$.

(e) $\lim _{t \rightarrow \infty} u_{i}\left(q(t), p(t), z_{i}(t)\right)=0$.

(f) $\lim _{t \rightarrow \infty}\left[A_{i}(q(t))+R_{i}(q(t))\right]=0$.

(g) $\lim _{t \rightarrow \infty} z_{i}(t) /\left\|z_{i}(t)\right\|=\chi / p_{\mathrm{f}}$.

Part (a) states that if $W(z(0), w(0))<\psi(0)$, then there are no collisions, which implies (F1). Part (b) states that every agent's velocity tends to $\chi$. Therefore, the difference in velocity between any 2 agents tends to zero, which implies (F2). Part (c) states that if the communication radius $r_{\mathrm{c}}$ is sufficiently large, then the maximum distance between any 2 agents is asymptotically bounded by $d(n-1)$, which implies (F3). Part (d) states that the derivative of $\psi_{\mathrm{s}}$ along the trajectories of (18)-(20) tends to 0 . Thus, the asymptotic configuration of agents is a critical point of $\psi_{\mathrm{s}}$. Furthermore, [10, Lemma 3] shows that if $r_{\mathrm{c}} / d$ is sufficiently small and the asymptotic configuration of the agents in $\mathcal{I}$ minimizes $\psi_{\mathrm{s}}$, then that configuration satisfies (F4). Part (e) states that the control tends to 0. Part (f) states that the sum of flock attraction and flock repulsion of each agent tends to 0. Part (g) states that the direction of $z_{i}(t)$ tends to the direction of the destination velocity $\chi$.

Part (a) of Theorem 1 provides a sufficient condition on $z(0)$ and $w(0)$ such that there are no collisions. If $q(0)$ is close to a flocking formation (i.e., a minimum of $\left.\psi_{\mathrm{s}}\right), p(0)$ is close to the destination velocity $\chi$ (i.e., $w(0) \approx 0)$, and $\chi^{\mathrm{T}} z_{i} \approx p_{\mathrm{f}}\left\|z_{i}\right\|$, then $W(z(0), w(0))$ is close to its minimum. In this case, $W(z(0), w(0))$ tends to satisfy $W(z(0), w(0))<\psi(0)$, and part (a) of Theorem 1 implies that there are no collisions. We note that the upper bound $\psi(0)$ in part (a) tends to increase as the repulsion parameter $b$ increases. Numerical testing suggests that for initial conditions $z(0)$ and $w(0)$, where $W(z(0), w(0))$ is not near its minimum, selecting the repulsion parameter $b$ sufficiently large tends to result in $W(z(0), w(0))$ and $\psi(0)$ that satisfy $W(z(0), w(0))<\psi(0)$; in this case, part (a) implies that there are no collisions. We also note that $W(z(0), w(0))<\psi(0)$ is a sufficient-but-not-necessary condition such that there are no collisions. In fact, numerical testing suggests that for many initial conditions $z(0)$ and $w(0)$, where $W(z(0), w(0)) \geq \psi(0)$, there are no collisions. 


\section{Destination-Seeking Analysis}

In this section, we analyze the asymptotic properties of (1), (2), and (18) without (A1) and (A2). We provide a sufficient condition such that agents approach a subset of the closed-loop equilibria of (1), (2), and (18). We also provide a sufficient condition such that agents approach their destinations asymptotically. Define $u(q, p) \triangleq\left[\begin{array}{lll}u_{1}^{\mathrm{T}}\left(q, p, \xi_{1}-q_{1}\right) & \cdots & u_{n}^{\mathrm{T}}\left(q, p, \xi_{n}-q_{i}\right)\end{array}\right]^{\mathrm{T}}$, where $u_{i}$ is given by (18).

The set of closed-loop equilibria of (1), (2), and (18) is

$$
\mathcal{E} \triangleq\left\{(q, p) \in \mathbb{R}^{n m} \times \mathbb{R}^{n m}: p=0 \text { and } u(q, 0)=0\right\} .
$$

Figure 3 shows 4 types of equilibria for $n=2$. In Figure $3(\mathrm{a}), q(t) \equiv \xi$, and all terms in $u$ are zero. In Figure 3(b), the sum of flock attraction and destination seeking is zero, that is, $\mu\left(\| z_{i}||\right) A_{i}(q)+\nu\left(\left\|z_{i}\right\|\right) z_{i}=0$, and all other terms in $u$ are zero. In Figure 3(c) and 3(d), the sum of flock repulsion and destination seeking is zero, that is, $R_{i}(q)+\nu\left(\left\|z_{i}\right\|\right) z_{i}=0$, and all other terms in $u$ are zero. For $n \geq 2$, the closed-loop system (1), (2), and (18) has equilibria that consist of combinations of the 4 types shown in Figure 3.

$$
\begin{aligned}
& q_{1}=\xi_{1} \\
& \bigotimes \\
& q_{2}=\xi_{2} \\
& \bigotimes \\
& \text { (a) } q_{i}=\xi_{i}
\end{aligned}
$$

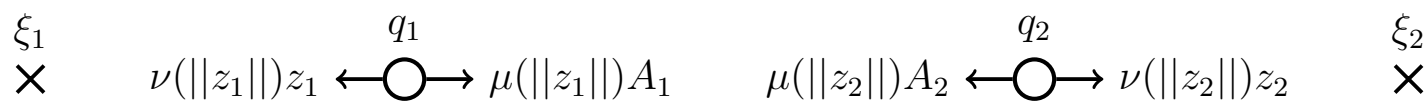

$$
\begin{aligned}
& \text { (b) } \mu\left(\left\|z_{i}\right\|\right) A_{i}(q)+\nu\left(\left\|z_{i}\right\|\right) z_{i}=0 \\
& R_{1} \stackrel{q_{1}}{\mathrm{\bigcirc}} \nu\left(\left\|z_{1}\right\|\right) z_{1} \quad \stackrel{\xi_{2}}{\mathrm{X}} \quad \nu\left(\| z_{2}||\right) z_{2} \stackrel{q_{2}}{\longleftarrow} \longrightarrow R_{2} \quad \text { × } \\
& \text { (c) } R_{i}(q)+\nu\left(\| z_{i}||\right) z_{i}=0
\end{aligned}
$$

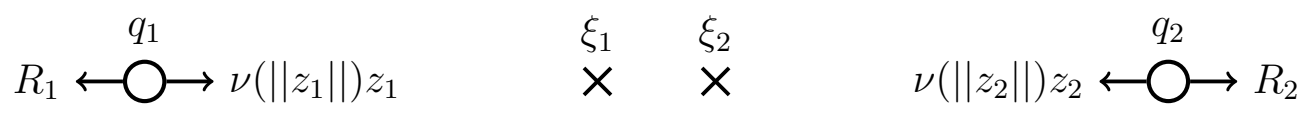

$$
\begin{aligned}
& \text { (d) } R_{i}(q)+\nu\left(\left\|z_{i}\right\|\right) z_{i}=0
\end{aligned}
$$

Figure 3: Four types of equilibria for $n=2$. Agents are shown by $\bigcirc$ s, and destinations are shown by $\times$ s. In (a), $q(t) \equiv \xi$, and all terms in $u$ are zero. In (b), the sum of flock attraction and destination seeking is zero, and all other terms in $u$ are zero. In (c) and (d), the sum of flock repulsion and destination seeking is zero, and all other terms in $u$ are zero.

Figure 3 shows that $(\xi, 0)$ is not the only closed-loop equilibria of $(1),(2)$, and (18). In 
fact, $(\xi, 0)$ may not be in $\mathcal{E}$. Define

$$
r_{\gamma} \triangleq \begin{cases}d, & \text { if } r_{\alpha} \in(0, d], \\ r_{\alpha}, & \text { if } r_{\alpha} \in\left(d, r_{\mathrm{c}}\right), \\ r_{\mathrm{c}}, & \text { if } r_{\alpha} \in\{0\} \cup\left[r_{\mathrm{c}}, \infty\right) .\end{cases}
$$

The radius $r_{\gamma}$ is such that if all agents are at their destinations and the destinations are at least $r_{\gamma}$ apart, then flock attraction, flock repulsion, and velocity consensus are 0 . The following result provides a sufficient condition such that $(\xi, 0) \in \mathcal{E}$. The proof is in Appendix $\mathrm{C}$.

Lemma 1. If, for all $(i, j) \in \mathcal{P},\left\|\xi_{j}-\xi_{i}\right\| \in\{d\} \cup\left[r_{\gamma}, \infty\right)$, then $(\xi, 0) \in \mathcal{E}$.

The following result provides a sufficient condition such that all agents asymptotically approach their destinations. This result is a consequence of Proposition 2, and the proof is in Appendix D.

Theorem 2. Consider the closed-loop system (1), (2), and (18), where, for all $(i, j) \in \mathcal{P}$, $\left\|\xi_{j}-\xi_{i}\right\| \geq r_{\gamma}$, and define $r_{\delta} \triangleq \frac{1}{2}\left(\min _{(i, j) \in \mathcal{P}}\left\|\xi_{j}-\xi_{i}\right\|-d\right) \geq 0$. Assume that there exists $t_{0} \geq 0$ such that, for all $t \geq t_{0}$ and all $i \in \mathcal{I}$, at least one of the following conditions holds:

(a) For all $j \in \mathcal{I} \backslash\{i\},\left\|q_{j}(t)-q_{i}(t)\right\|>r_{\mathrm{c}}$.

(b) $\left\|z_{i}(t)\right\|<\min \left\{r_{\alpha}, r_{\delta}\right\}$.

Then, $\lim _{t \rightarrow \infty} q(t)=\xi$ and $\lim _{t \rightarrow \infty} p(t)=0$.

Theorem 2 states that if there exists a time after which all agents are greater than $r_{\mathrm{c}}$ apart from other agents or less than $\min \left\{r_{\alpha}, r_{\delta}\right\}$ from their destinations, then all agents asymptotically approach their destinations. The assumption that there exists a time such that all agents are sufficiently far from other agents or sufficiently close to their destinations precludes agents from converging to equilibria, where nonzero flock attraction or repulsion cancel nonzero destination seeking. Examples of these types of equilibria are shown in Figure 3(b)-(d). If flock attraction is sufficiently small relative to destination seeking, then numerical simulations suggest that the assumption of Theorem 2 holds. To decrease flock attraction relative to destination seeking, we decrease $a$ or increase $\gamma$ and $\omega_{\mathrm{n}}$.

We now use Lyapunov-like analysis to examine the asymptotic properties of (1), (2), and (18) without the assumption that, for sufficiently large $t \geq 0$, all agents are greater than $r_{\mathrm{c}}$ apart from other agents or less than $\min \left\{r_{\alpha}, r_{\beta}\right\}$ from their destinations. Consider the Lyapunov-like function $V: \mathbb{R}^{n m} \times \mathbb{R}^{n m} \rightarrow[0, \infty)$ defined by

$$
V(q, p) \triangleq \psi_{\mathrm{s}}(q)+\sum_{i \in \mathcal{I}} \int_{0}^{\left\|\xi_{i}-q_{i}\right\|} \lambda \nu(\lambda) \mathrm{d} \lambda+\frac{1}{2}\|p\|^{2}
$$

If, for all $(i, j) \in \mathcal{P},\left\|\xi_{j}-\xi_{i}\right\|=d, q_{i}=\xi_{i}$, and $p_{i}=0$, then $V(q, p)=0$; otherwise, $V(q, p)>0$. Note that $V(q, p)$ has multiple minimizers.

The following result provides a sufficient condition such that $(q(t), p(t)) \equiv(\xi, 0)$ is a locally asymptotically equilibrium of (1), (2), and (18), and the agents do not collide. This result also shows that $(q(t), p(t))$ converges to a subset of $\mathcal{E}$. The proof is in Appendix D. 
Theorem 3. Consider the closed-loop system (1), (2), and (18), and let $r_{\alpha}=r_{\beta}=0$. Then, the following statements hold:

(a) Assume that, for all $(i, j) \in \mathcal{P},\left\|\xi_{j}-\xi_{i}\right\|>r_{\mathrm{c}}$. Then, $(q(t), p(t)) \equiv(\xi, 0)$ is a locally asymptotically stable equilibrium of (1), (2), and (18).

(b) For all $q(0) \in \mathbb{R}^{n m}$ and all $p(0) \in \mathbb{R}^{n m},(q(t), p(t))$ converges to the set $\mathcal{M} \triangleq\{(q, p) \in$ $\mathcal{E}: V(q, p) \leq V(q(0), p(0))\}$.

(c) If $V(q(0), p(0))<\psi(0)$, then, for all $(i, j) \in \mathcal{P}$ and all $t \geq 0, q_{j}(t) \neq q_{i}(t)$.

Part (a) states that if all destinations are at least $r_{\mathrm{c}}$ apart from one another, then $(q(t), p(t)) \equiv(\xi, 0)$ is a locally asymptotically stable equilibrium of (1), (2), and (18). Part (b) states that, for all initial conditions, $(q, p)$ converges to $\mathcal{M}$, which is a subset of $\mathcal{E}$. Therefore, all agents tend to zero velocity and zero control. Part (c) states that if $V(q(0), p(0))<\psi(0)$, then there are no collisions. Part (c) provides a sufficient condition such that there are no collisions. The condition in part (c) is not necessary.

\section{Numerical Examples}

In this section, we present examples that demonstrate the control (18). Unless otherwise stated, $d=15 \mathrm{~m}, r_{\mathrm{c}}=30 \mathrm{~m}, r_{\alpha}=r_{1}=25 \mathrm{~m}, r_{\beta}=r_{2}=50 \mathrm{~m}, a=0.33, b=3.5, h=0.2$, $\epsilon=0.1, \omega_{\mathrm{n}}=0.5 \mathrm{rad} / \mathrm{s}, p_{\mathrm{f}}=20 \mathrm{~m} / \mathrm{s}, \gamma=5 \mathrm{~m} / \mathrm{s}^{2}$, and $\mu$ is given by (11). Let $\hat{e}_{k} \in \mathbb{R}^{1 \times m}$ be the $k$ th row of the $m \times m$ identity matrix. Flocking condition (F1) states that for all $t \geq 0$ and all $(i, j) \in \mathcal{P}, q_{i}(t) \neq q_{j}(t)$. However, most applications require that $\left\|q_{j}(t)-q_{i}(t)\right\|>\delta_{\mathrm{c}}$, where $\delta_{\mathrm{c}}>0$ is a collision threshold. For the following examples, we consider $\delta_{\mathrm{c}}=0.5 \mathrm{~m}$.

Example 1. This example shows the effect of $\gamma$. Consider $n=3$ agents, where $m=2$, $q_{1}(0)=\left[\begin{array}{ll}0 & 8\end{array}\right]^{\mathrm{T}} \mathrm{m}, \xi_{1}=\left[\begin{array}{ll}1500 & 50\end{array}\right]^{\mathrm{T}} \mathrm{m}, q_{2}(0)=\left[\begin{array}{ll}0 & 0\end{array}\right]^{\mathrm{T}} \mathrm{m}, \xi_{2}=\left[\begin{array}{ll}2600 & 20\end{array}\right]^{\mathrm{T}} \mathrm{m}$, $q_{3}(0)=\left[\begin{array}{ll}0 & -24\end{array}\right]^{\mathrm{T}} \mathrm{m}, \xi_{3}=\left[\begin{array}{ll}2700 & -55\end{array}\right]^{\mathrm{T}} \mathrm{m}$, and $p_{1}(0)=p_{2}(0)=p_{3}(0)=\left[\begin{array}{ll}0 & 0\end{array}\right]^{\mathrm{T}} \mathrm{m} / \mathrm{s}$. We consider 2 cases: $\gamma=2 \mathrm{~m} / \mathrm{s}^{2}$ and $\gamma=9 \mathrm{~m} / \mathrm{s}^{2}$. Figures 4 and 5 show that as $\gamma$ decreases from $9 \mathrm{~m} / \mathrm{s}^{2}$ to $2 \mathrm{~m} / \mathrm{s}^{2}$, the agents are close together over a longer distance, take longer to approach their destinations, and tend to $p_{\mathrm{f}}=20 \mathrm{~m} / \mathrm{s}$ more slowly. In both cases, for all time the minimum interagent distance is above the collision threshold, that is, for all $t \geq 0$, $\min _{(i, j) \in \mathcal{P}}\left\|q_{j}(t)-q_{i}(t)\right\|>\delta_{\mathrm{c}}=0.5 \mathrm{~m}$. Thus, the agents achieve (O1) and (O2).

Example 2. This example considers a group of autonomous vehicles on a highway. Consider $n=6$ agents, where $m=2, r_{\mathrm{c}}=70 \mathrm{~m}$, and $a=0.8$. Each agent starts with zero velocity, and the control (18) is turned on once another agent is less than $0.75 r_{\mathrm{c}}$ away. Figure 6 shows the trajectories of each agent, where the time next to each agent's initial position indicates the time at which it starts moving. While agents are far from their destinations, they flock with neighboring agents. As agents approach their destinations, they leave the flock, and the remaining agents form a new flock. Figure 7 shows the minimum interagent distance $\min _{(i, j) \in \mathcal{P}}\left\|q_{j}-q_{i}\right\|$ and the maximum control magnitude $\max _{i \in \mathcal{I}}\left\|u_{i}\right\|$. The minimum interagent distance is always greater than the collision threshold $\delta_{\mathrm{c}}$ and reaches a minimum of approximately $d=15 \mathrm{~m}$. The maximum control magnitude reaches a maximum of approximately $6 \mathrm{~m} / \mathrm{s}^{2}$ at $t=40 \mathrm{~s}$. 


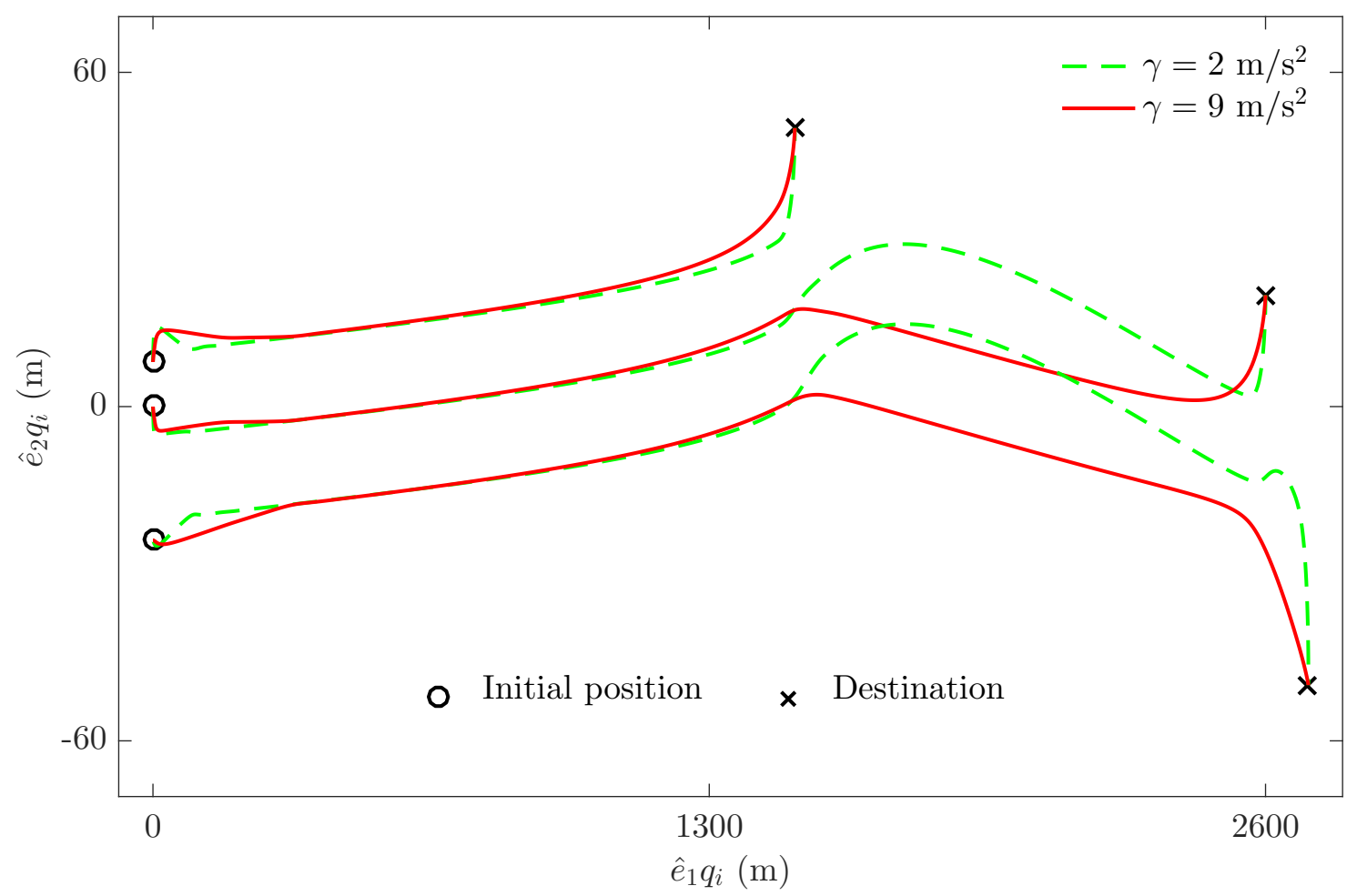

Figure 4: Trajectories of $n=3$ agents for $\gamma=2 \mathrm{~m} / \mathrm{s}^{2}$ and $9 \mathrm{~m} / \mathrm{s}^{2}$. As $\gamma$ decreases from $9 \mathrm{~m} / \mathrm{s}^{2}$ to $2 \mathrm{~m} / \mathrm{s}^{2}$, agents are close together over a longer distance. The agents achieve (O1) and (O2).
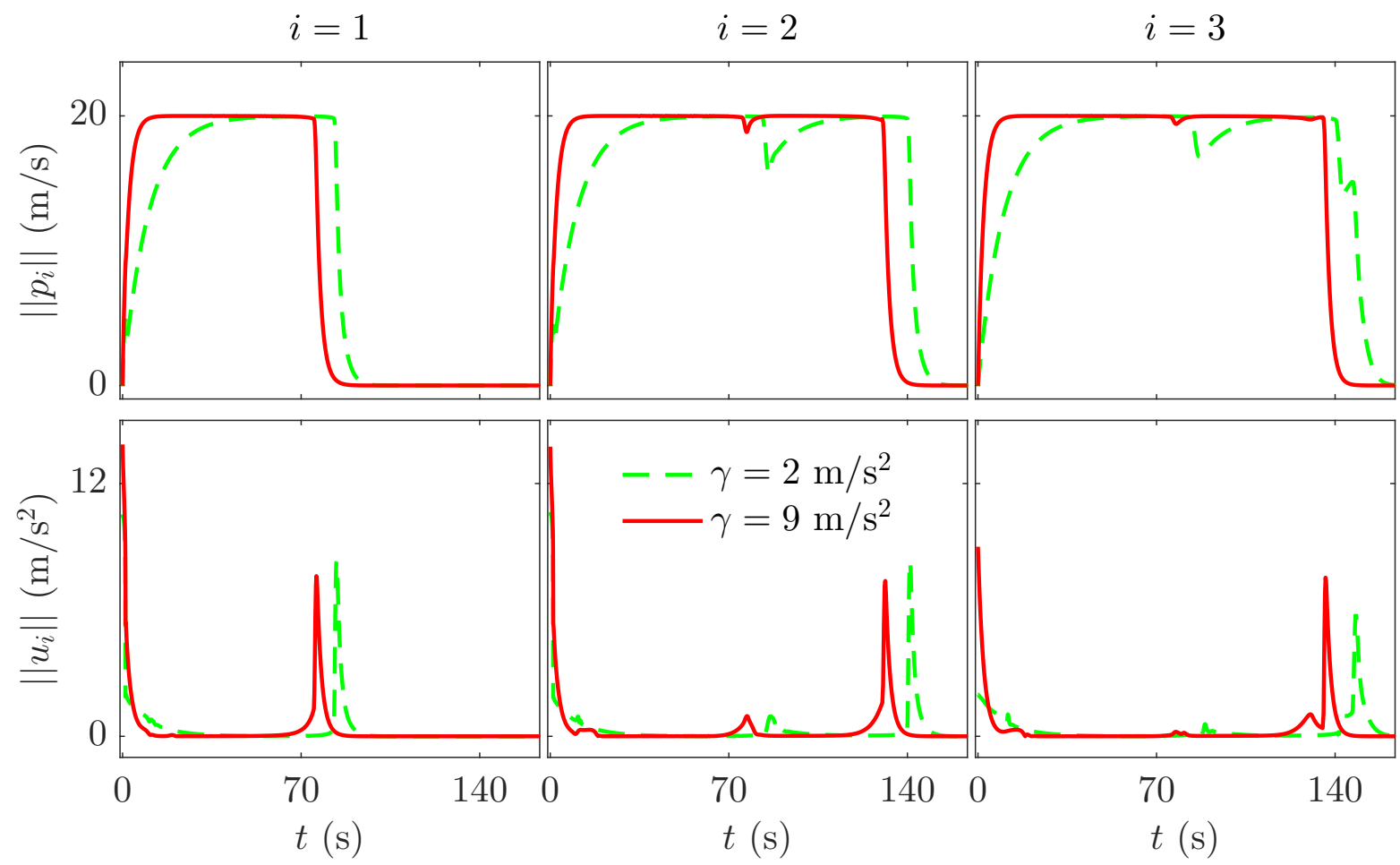

Figure 5: Speed and magnitude of control of $n=3$ agents for $\gamma=2 \mathrm{~m} / \mathrm{s}^{2}$ and $9 \mathrm{~m} / \mathrm{s}^{2}$. As $\gamma$ decreases from 9 $\mathrm{m} / \mathrm{s}^{2}$ to $2 \mathrm{~m} / \mathrm{s}^{2}$, agents takes longer to approach their destinations and tend to $p_{\mathrm{f}}=20 \mathrm{~m} / \mathrm{s}$ more slowly. The agents achieve (O1) and (O2). 


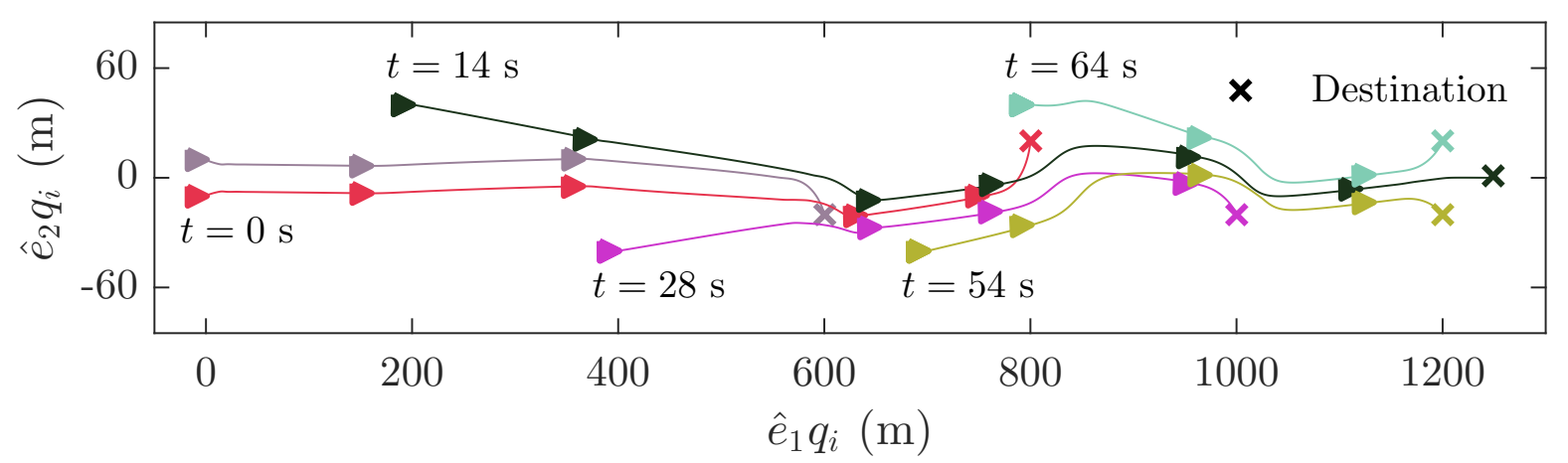

Figure 6: A group of $n=6$ agents that flock before they reach their destinations. Each agent starts with zero velocity, and the control (18) is applied once another agent is less than $0.75 r_{\mathrm{c}}$ away. The time next to each agent's initial position indicates the time at which it starts moving. While agents are far from their destinations, they flock with neighboring agents. As agents approach their destinations, they leave the flock, and the remaining agents form a new flock.
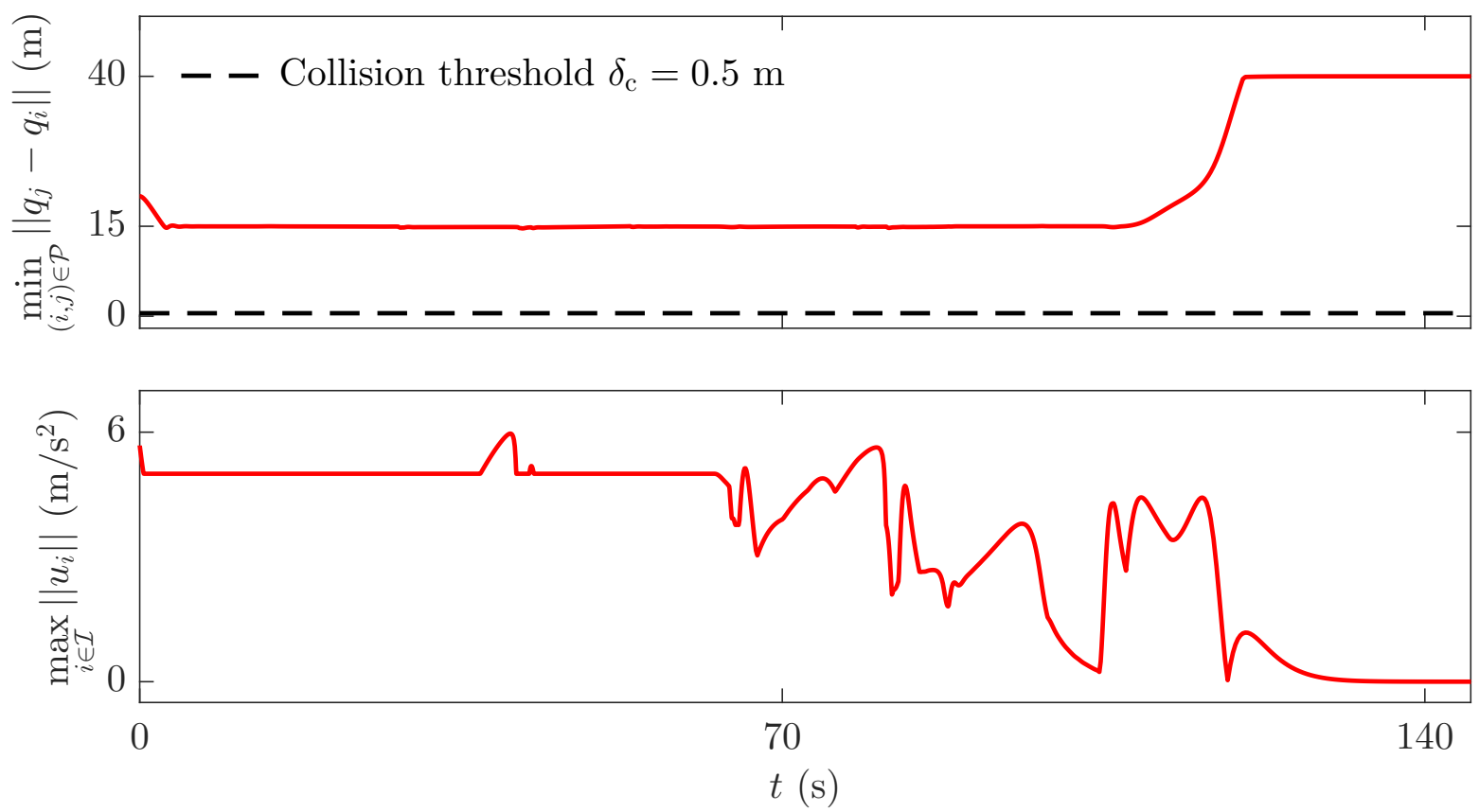

Figure 7: For the trajectory shown in Figure 6, the minimum interagent distance $\min _{(i, j) \in \mathcal{P}}\left\|q_{j}-q_{i}\right\|$ is always greater than the collision threshold $\delta_{\mathrm{c}}$ and reaches a minimum of approximately $d=15 \mathrm{~m}$, and the maximium control magnitude $\max _{i \in \mathcal{I}}\left\|u_{i}\right\|$ reaches a maximum of approximately $6 \mathrm{~m} / \mathrm{s}^{2}$ at $t=40 \mathrm{~s}$. 
Example 3. Consider $n=20$ agents, where $m=2$, the initial velocities are all zero, and 10 agents are distributed near $\left[\begin{array}{ll}0 & 300\end{array}\right]^{\mathrm{T}} \mathrm{m}$, while the other 10 agents are distributed near $\left[\begin{array}{ll}0 & -300\end{array}\right]^{\mathrm{T}} \mathrm{m}$. Figure 8 shows that initially 2 flocks are formed. Then, each flock splits into 2 flocks based on agents' destinations. Next, 2 of the flocks cross without collisions or fragmentation. Finally, all agents exit the flocks and approach their destinations. Figure 9 shows that the minimum interagent distance is always greater than the collision threshold $\delta_{\mathrm{c}}$ is at a minimum of approximately $1 \mathrm{~m}$ at $t=0 \mathrm{~s}$, and the maximium control magnitude is at a maximum of approximately of $44 \mathrm{~m} / \mathrm{s}^{2}$ at $t=0 \mathrm{~s}$. Thus, the agents achieve (O1) and (O2).

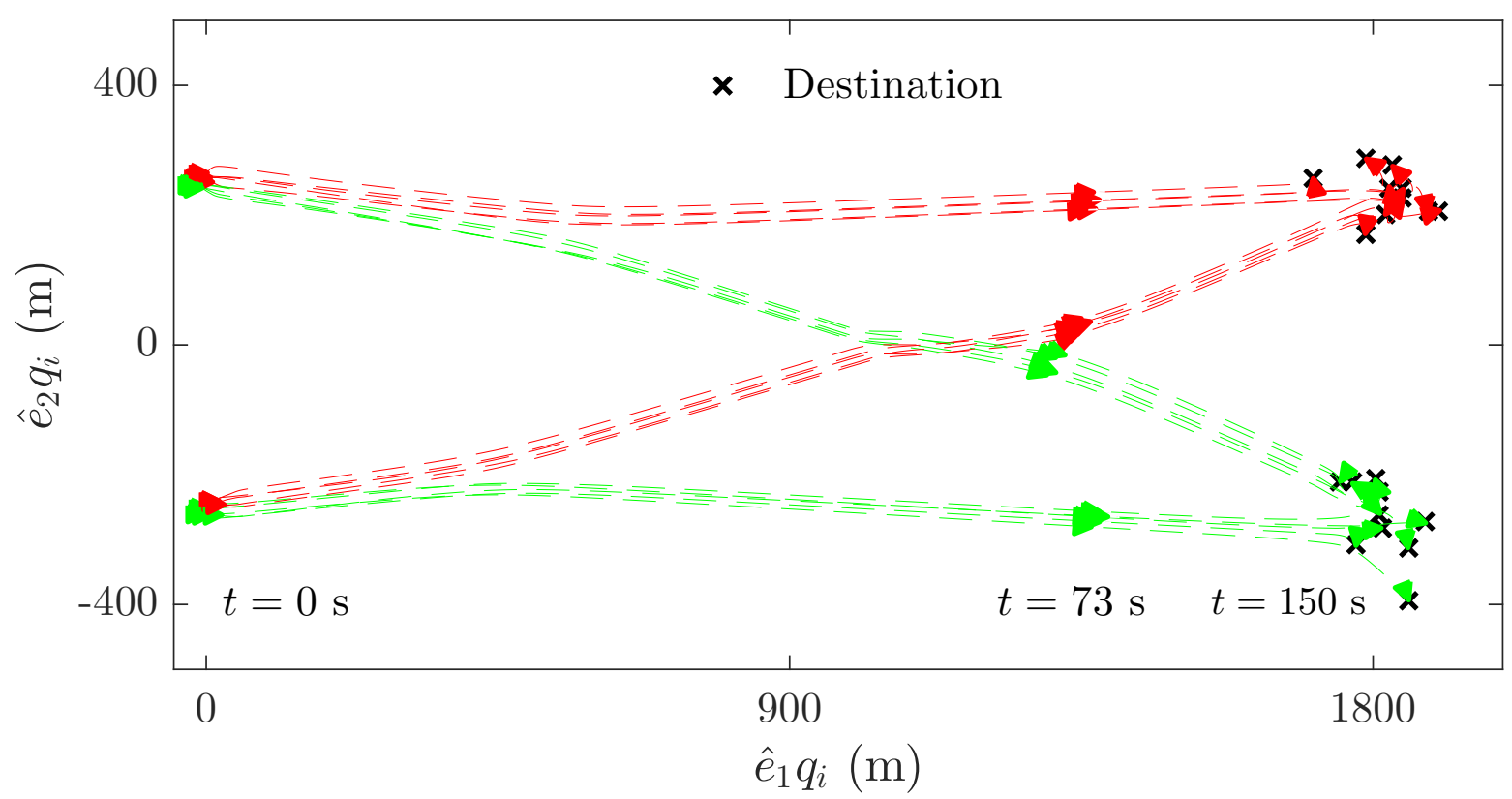

Figure 8: A group of $n=20$ agents that flock before they reach their destinations. Initially 2 flocks are formed. Then, each of those flocks splits into 2 flocks based on the agents' destinations. Next, 2 of the flocks cross without collisions or fragmentation. Finally, all agents exit the flocks and approach their destinations. Thus, the agents achieve (O1) and (O2).

Example 4. Consider $n=20$ agents, where $m=3$, all agents are normally distributed about $\left[\begin{array}{lll}0 & 0 & 200\end{array}\right]^{\mathrm{T}} \mathrm{m}$ with variance $225 \mathrm{~m}^{2}$, and the initial velocities are normally distributed about $\left[\begin{array}{lll}0 & 0 & 0\end{array}\right]^{\mathrm{T}} \mathrm{m} / \mathrm{s}$ with variance $16 \mathrm{~m}^{2} / \mathrm{s}^{2}$. Figure 10 shows that the agents flock by $t=26 \mathrm{~s}$. Then, the agents exit the flock and approach their destinations. Figure 11 shows that the minimum interagent distance is always greater than the collision threshold $\delta_{\mathrm{c}}$ and is at a minimum of approximately $4.4 \mathrm{~m}$ at $t=0 \mathrm{~s}$, and the maximium control magnitude reaches a maximum of approximately $46 \mathrm{~m} / \mathrm{s}^{2}$ at $t=0.6 \mathrm{~s}$. Thus, the agents achieve (O1) and (O2).

\section{Conclusions}

We presented the flocking-and-destination-seeking control (18) for a set of $n$ agents with double-integrator dynamics and potentially unique destinations. The control (18) includes 

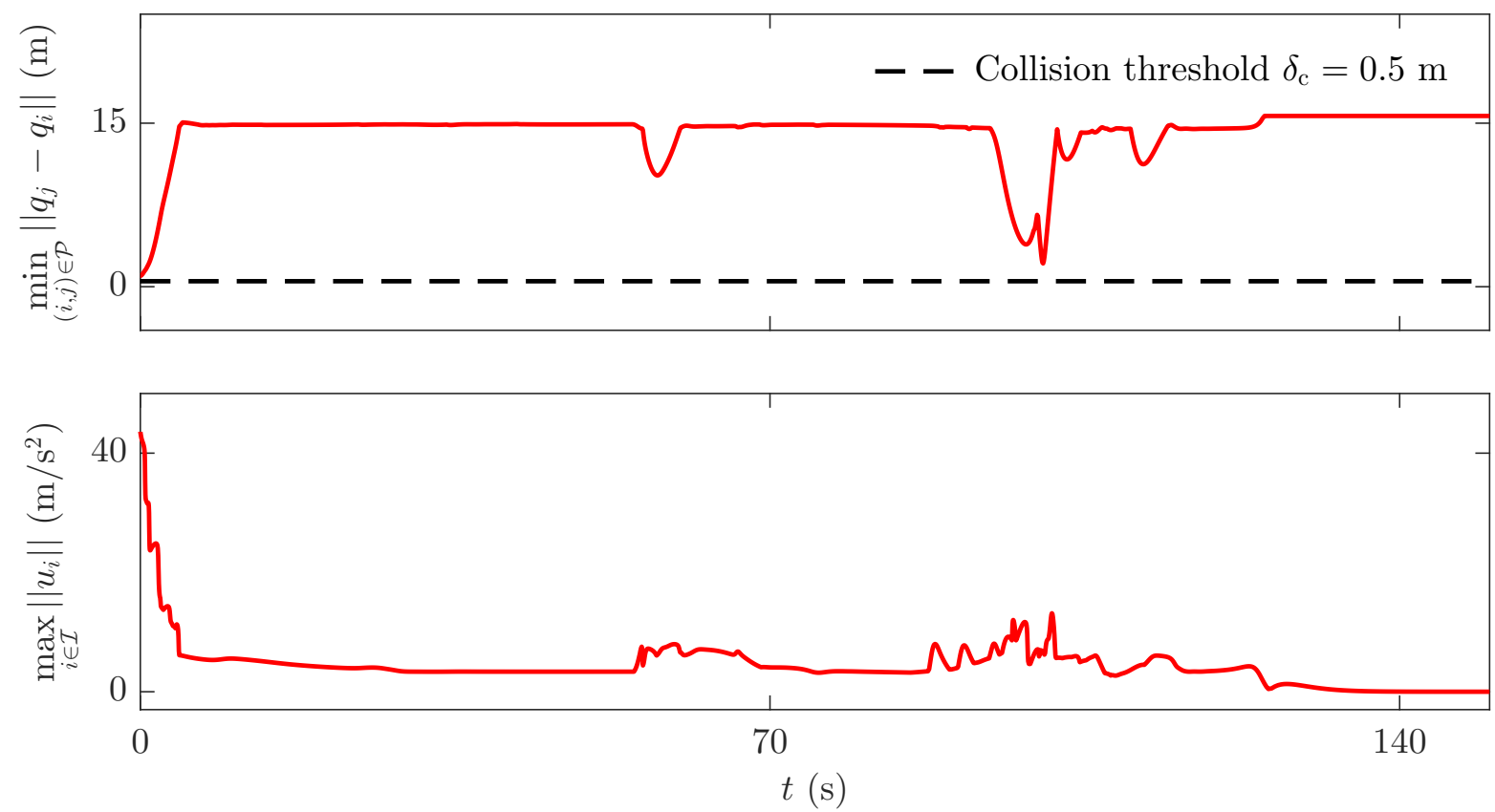

Figure 9: For the trajectory shown in Figure 8, the minimum interagent distance is always greater than the collision threshold $d$ and is at a minimum of approximately $1 \mathrm{~m}$ at $t=0 \mathrm{~s}$, and the maximium control magnitude is at a maximum of approximately $44 \mathrm{~m} / \mathrm{s}^{2}$ at $t=0 \mathrm{~s}$.

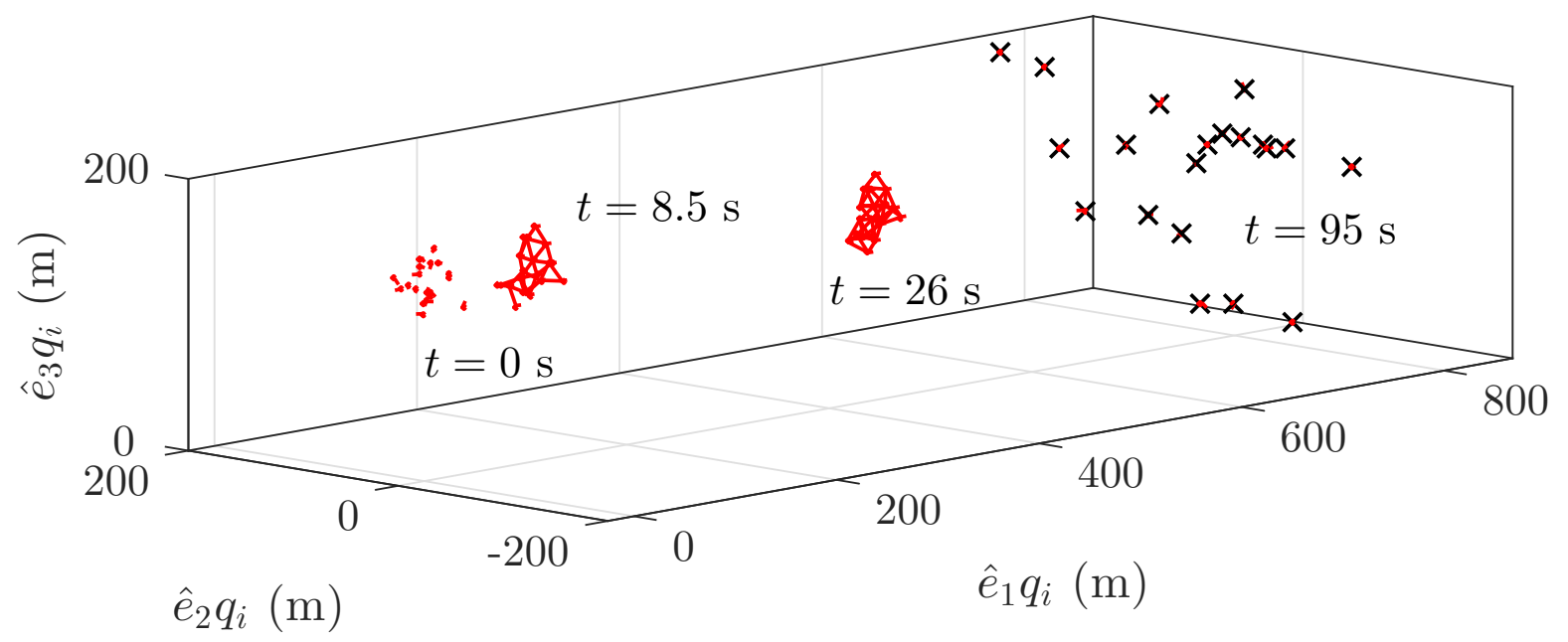

Figure 10: A group of $n=20$ agents that flock before they reach their destinations. The agents flock by $t=26 \mathrm{~s}$. Then, the agents exit the flock and approach their destinations. The agents achieve (O1) and (O2). The dots indicate agent positions, while the lines connecting dots indicate that the 2 agents are approximately $d=15 \mathrm{~m}$ apart. 

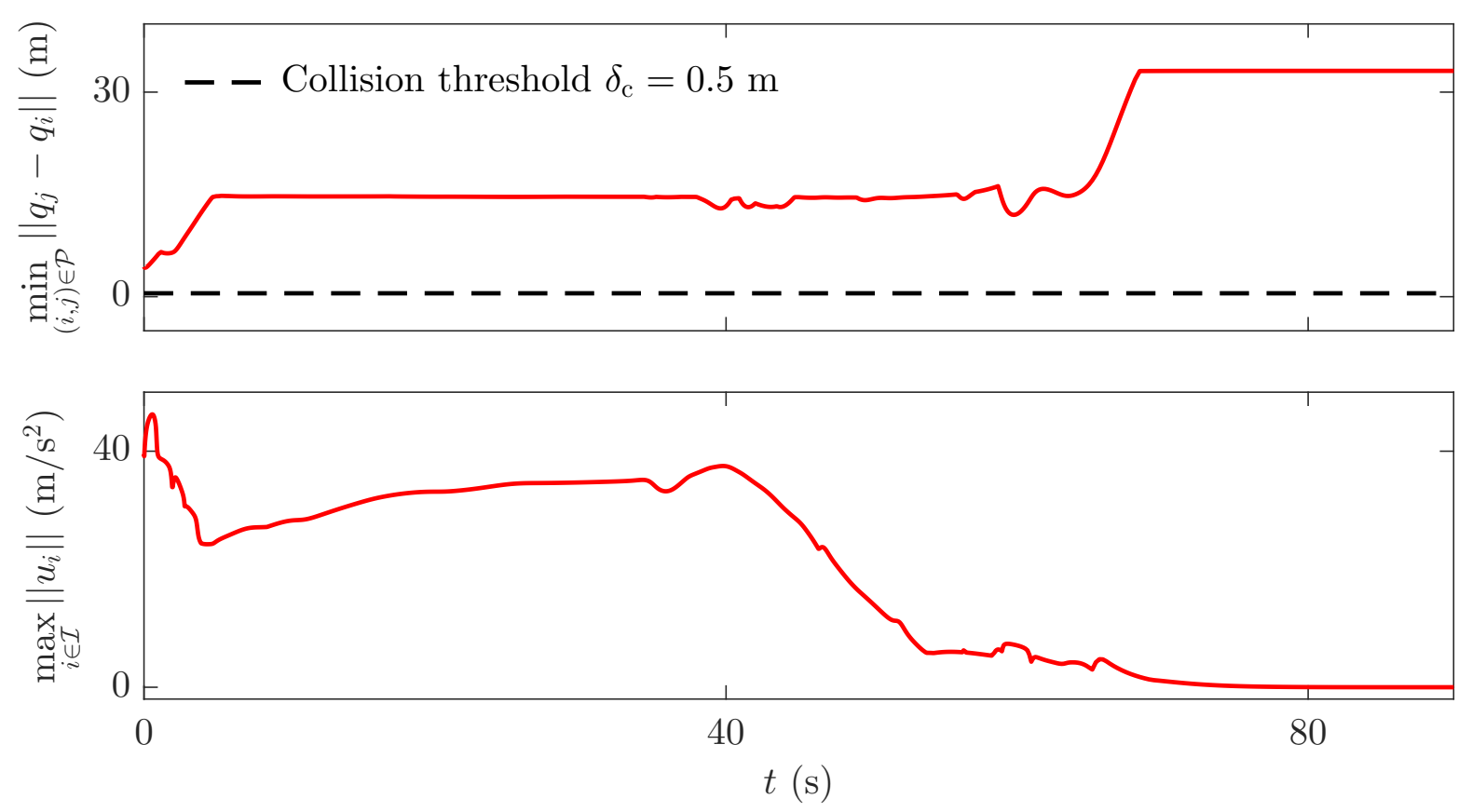

Figure 11: For the trajectory shown in Figure 10, the minimum interagent distance is always greater than the collision threshold $\delta_{\mathrm{c}}$ and is at a minimum of approximately $4.4 \mathrm{~m}$ at $t=0 \mathrm{~s}$, and the maximium control magnitude reaches a maximum of approximately $46 \mathrm{~m} / \mathrm{s}^{2}$ at $t=0.6 \mathrm{~s}$.

terms that induce flocking as well as terms that drive each agent towards its destination. The control is decentralized and does not require a centralized flock leader. The control accomplishes 2 objectives: (i) if an agent is far from its destination, then that agent flocks with nearby agents, and (ii) if an agent is close to its destination, then that agent approaches its destination.

\section{Acknowledgments}

This work is supported in part by the National Science Foundation (award number OIA-1539070) and the National Aeronautics and Space Administration (award number NNX10AL96) through NASA Kentucky Space Grant.

\section{Appendix A. Proof of Proposition 2}

Proof of Proposition 2. To show (a), consider the Lyapunov function $V_{1}: \mathbb{R}^{n m} \times$ $\mathbb{R}^{n m} \rightarrow[0, \infty)$ defined by

$$
V_{1}(q, p) \triangleq \sum_{i \in \mathcal{I}} \int_{0}^{\left\|\xi_{i}-q_{i}\right\|} \lambda \nu(\lambda) \mathrm{d} \lambda+\frac{1}{2}\|p\|^{2}
$$

which is radially unbounded in $q$ and $p$. If $q=\xi$ and $p=0$, then $V_{1}(q, p)=0$; otherwise, $V_{1}(q, p)>0$. The derivative of $V_{1}(q, p)$ along the trajectories of (1), (2), and (16) is

$$
\dot{V}_{1}(q, p) \triangleq \sum_{i \in \mathcal{I}} \frac{\partial V_{1}(q, p)}{\partial q_{i}} p_{i}+\frac{\partial V_{1}(q, p)}{\partial p_{i}} u_{i}\left(z_{i}, p_{i}\right)
$$




$$
=\sum_{i \in \mathcal{I}}\left(\frac{\partial}{\partial q_{i}}\left[\int_{0}^{\left\|\xi_{i}-q_{i}\right\|} \lambda \nu(\lambda) \mathrm{d} \lambda\right]+\nu\left(\left\|z_{i}\right\|\right) z_{i}^{\mathrm{T}}+\kappa\left(\left\|z_{i}\right\|\right) p_{i}^{\mathrm{T}}\right) p_{i} .
$$

Define $\nu^{\prime}(\eta) \triangleq \nu(\sqrt{\eta})$, and it follows that $\int_{0}^{\left\|\xi_{i}-q_{i}\right\|} \lambda \nu(\lambda) \mathrm{d} \lambda=\frac{1}{2} \int_{0}^{\left\|\xi_{i}-q_{i}\right\|^{2}} \nu^{\prime}(\tau) \mathrm{d} \tau$, where $\tau=\lambda^{2}$ and $\mathrm{d} \tau=2 \lambda \mathrm{d} \lambda$. Thus, $\frac{\partial}{\partial q_{i}}\left[\int_{0}^{\left\|\xi_{i}-q_{i}\right\|} \lambda \nu(\lambda) \mathrm{d} \lambda\right]=\frac{\partial}{\partial q_{i}}\left[\frac{1}{2} \int_{0}^{\left\|\xi_{i}-q_{i}\right\|^{2}} \nu^{\prime}(\tau) \mathrm{d} \tau\right]=-\nu\left(\left\|\xi_{i}-q_{i}\right\|\right)\left[\xi_{i}-\right.$ $\left.q_{i}\right]^{\mathrm{T}}=-\nu\left(\left\|z_{i}\right\|\right) z_{i}^{\mathrm{T}}$. Therefore, $\dot{V}_{1}(q, p)=\sum_{i \in \mathcal{I}} \kappa\left(\left\|z_{i}\right\|\right) p_{i}^{\mathrm{T}} p_{i}$, which is nonpositive because $\kappa<0$.

Define $\mathcal{S} \triangleq\left\{(q, p) \in \mathbb{R}^{n m} \times \mathbb{R}^{n m}: \dot{V}_{1}(q, p)=0\right\}$. Since $\dot{V}_{1}(q, p)=0$ implies that $p=0$, it follows that $\mathcal{S}=\left\{(q, p) \in \mathbb{R}^{n m} \times \mathbb{R}^{n m}: p=0\right\}$. Define $E_{i} \triangleq\left[\begin{array}{lll}0_{m \times m(i-1)} & I_{m} & 0_{m \times m(n-i)}\end{array}\right] \in$ $\mathbb{R}^{m \times n m}$, where $0_{l_{1} \times l_{2}}$ is the $l_{1} \times l_{2}$ matrix of zeros, and $I_{m}$ is the $m \times m$ identity matrix. Let $\tilde{q}:[0, \infty) \rightarrow \mathbb{R}^{n m}$ and $\tilde{p}:[0, \infty) \rightarrow \mathbb{R}^{n m}$ be such that, for all $t \geq 0,(\tilde{q}(t), \tilde{p}(t)) \in \mathcal{S}$ and, for all $i \in \mathcal{I}, \tilde{q}_{i}(t) \triangleq E_{i} \tilde{q}(t)$ and $\tilde{p}_{i}(t) \triangleq E_{i} \tilde{p}(t)$ satisfy (1), (2), and (16). Since, for all $t \geq 0$, $(\tilde{q}(t), \tilde{p}(t)) \in \mathcal{S}$, it follows that $\tilde{p}(t) \equiv 0$. Furthermore, since $z_{i}=\xi_{i}-q_{i}$, it follows from (2) and (16) that

$$
0 \equiv \dot{\tilde{p}}_{i}(t) \equiv \tilde{p}_{i}(t)+u_{i}\left(\xi_{i}-\tilde{q}_{i}(t), \tilde{p}_{i}(t)\right) \equiv \nu\left(\left\|\xi_{i}-\tilde{q}_{i}(t)\right\|\right)\left[\xi_{i}-\tilde{q}_{i}(t)\right]
$$

which implies that $\tilde{q}_{i}(t) \equiv \xi_{i}$ because $\nu>0$. Thus, $(\tilde{q}(t), \tilde{p}(t)) \equiv(\xi, 0)$ is the only solution of (1), (2), and (16) such that, for all $t \geq 0,(\tilde{q}(t), \tilde{p}(t)) \in \mathcal{S}$. Therefore, [26, Corollary 4.2] implies that $(q(t), p(t)) \equiv(\xi, 0)$ is a globally asymptotically stable equilibrium of $(1),(2)$, and (16), which confirms (a).

To show $(\mathrm{b})$, since $(q(t), p(t)) \equiv(\xi, 0)$ is a globally asymptotically stable equilibrium of $(1)$, (2), and (16), it follows that there exists $t_{1}>0$ such that, for all $t \geq t_{1},\left\|z_{i}(t)\right\| \leq r_{1}$. Thus, (1), (2), and (14)-(16) imply that, for all $t \geq t_{1}, q_{i}(t)$ satisfies $\ddot{q}_{i}(t)+2 \zeta \omega_{\mathrm{n}} \dot{q}_{i}(t)+\omega_{\mathrm{n}}^{2} q_{i}(t)=\omega_{\mathrm{n}}^{2} \xi_{i}$, which confirms (b).

To show (c), assume $\left\|z_{i}(0)\right\|>r_{2}$. Therefore, there exists $t_{0}>0$ such that, for all $t \in\left[0, t_{0}\right),\left\|z_{i}(t)\right\|>r_{2}$, and it follows from (14)-(16) that, for all $t \in\left[0, t_{0}\right), u_{i}\left(q_{i}(t), p_{i}(t)\right)=$ $\gamma z_{i}(t) /\left\|z_{i}(t)\right\|-\gamma p_{i}(t) / p_{\mathrm{f}}$, which confirms (c).

Part $(\mathrm{d})$ follows from direct calculation.

\section{Appendix B. Proof of Theorem 1}

Proof of Theorem 1. Since (A2) holds, it follows from (M1) that, for all $t \geq 0$, $\mu\left(\left\|z_{i}(t)\right\|\right)=1$, which implies that the derivative of $W(z, w)$ along the trajectories of $(18)-(20)$ is

$$
\begin{aligned}
\dot{W}(z, w) \triangleq & \frac{\partial W(z, w)}{\partial z} w-\frac{\partial W(z, w)}{\partial w} g(q, p, z) \\
= & \sum_{i \in \mathcal{I}}\left(\frac{\partial}{\partial z_{i}}\left[\sum_{k \in \mathcal{I}} \gamma\left\|z_{k}\right\|-\frac{\gamma}{p_{\mathrm{f}}} \chi^{\mathrm{T}} z_{k}\right]+\frac{\partial \psi_{\mathrm{s}}(z)}{\partial z_{i}}-A_{i}^{\mathrm{T}}(q)-R_{i}^{\mathrm{T}}(q)-C_{i}^{\mathrm{T}}(q, p)\right. \\
& \left.\quad-\nu\left(\| z_{i}||\right) z_{i}^{\mathrm{T}}-\kappa\left(\left\|z_{i}\right\|\right) p_{i}^{\mathrm{T}}\right) w_{i},
\end{aligned}
$$

where $g(q, p, z) \triangleq\left[\begin{array}{lll}u_{1}^{\mathrm{T}}\left(q, p, z_{1}\right) & \cdots & u_{n}^{\mathrm{T}}\left(q, p, z_{n}\right)\end{array}\right]^{\mathrm{T}}$, where $u_{i}$ is given by (18).

Since (A1) holds, it follows that, for all $(i, j) \in \mathcal{P}, q_{j}-q_{i}=z_{i}-z_{j}$, which implies that $\psi\left(\left\|q_{j}-q_{i}\right\|_{\epsilon}\right)=\psi\left(\left\|z_{i}-z_{j}\right\|_{\epsilon}\right)$, and hence, $\psi_{\mathrm{s}}(q)=\psi_{\mathrm{s}}(z)$. Thus, using (4), (8), (9), (21), and 
(22), we obtain that, for all $i \in \mathcal{I}$,

$$
\begin{aligned}
\frac{\partial \psi_{\mathrm{s}}(z)}{\partial z_{i}}=-\frac{\partial \psi_{\mathrm{s}}(q)}{\partial q_{i}} & =-\frac{\partial}{\partial q_{i}}\left[\sum_{k=1}^{n-1} \sum_{j=k+1}^{n} \psi\left(\left\|q_{j}-q_{k}\right\|_{\epsilon}\right)\right] \\
& =-\sum_{j \in \mathcal{I} \backslash\{i\}} \frac{\partial \psi\left(\left\|q_{j}-q_{i}\right\|_{\epsilon}\right)}{\partial q_{i}} \\
& =\sum_{j \in \mathcal{I} \backslash\{i\}} \rho_{h}\left(\frac{\left\|q_{j}-q_{i}\right\|_{\epsilon}}{\left\|r_{\mathrm{c}}\right\|_{\epsilon}}\right) \Phi^{\mathrm{T}}\left(q_{j}-q_{i}\right) \\
& =A_{i}^{\mathrm{T}}(q)+R_{i}^{\mathrm{T}}(q) .
\end{aligned}
$$

Define $\mathcal{D} \triangleq\left\{(z, w) \in \mathbb{R}^{n m} \times \mathbb{R}^{n m}\right.$ : for all $i \in \mathcal{I},\left\|z_{i}\right\|>r_{2}$, where $\left.z=\left[\begin{array}{ccc}z_{1}^{\mathrm{T}} & \cdots & z_{n}^{\mathrm{T}}\end{array}\right]^{\mathrm{T}}\right\}$, and it follows from (14) and (15) that, for all $(z, w) \in \mathcal{D}$ and all $i \in \mathcal{I}$,

$$
\begin{aligned}
\left(\frac{\partial}{\partial z_{i}}\left[\sum_{k \in \mathcal{I}} \gamma\left\|z_{k}\right\|-\frac{\gamma}{p_{\mathrm{f}}} \chi^{\mathrm{T}} z_{k}\right]-\nu\left(\left\|z_{i}\right\|\right) z_{i}^{\mathrm{T}}-\kappa\left(\left\|z_{i}\right\|\right) p_{i}^{\mathrm{T}}\right) w_{i}= & {\left[\frac{\gamma}{\left\|z_{i}\right\|} z_{i}-\frac{\gamma}{p_{\mathrm{f}}} \chi\right]^{\mathrm{T}} w_{i}-\frac{\gamma}{\left\|z_{i}\right\|} z_{i}^{\mathrm{T}} w_{i} } \\
& +\frac{\gamma}{p_{\mathrm{f}}} p_{i}^{\mathrm{T}} w_{i} \\
= & -\frac{\gamma}{p_{\mathrm{f}}}\left\|w_{i}\right\|^{2} .
\end{aligned}
$$

Since, for all $(i, j) \in \mathcal{P}, z_{i}-z_{j}=q_{j}-q_{i}$ and $w_{i}-w_{j}=p_{j}-p_{i}$, it follows from (10) that, for all $(z, w) \in \mathcal{D}$,

$$
\begin{aligned}
\sum_{i \in \mathcal{I}} C_{i}^{\mathrm{T}}(q, p) w_{i} & =\sum_{i \in \mathcal{I}} \sum_{j \in \mathcal{I} \backslash\{i\}} \rho_{h}\left(\frac{\left\|q_{j}-q_{i}\right\|_{\epsilon}}{\left\|r_{\mathrm{c}}\right\|_{\epsilon}}\right)\left[w_{i}^{\mathrm{T}} w_{i}-w_{j}^{\mathrm{T}} w_{i}\right] \\
& =\frac{1}{2} \sum_{(i, j) \in \mathcal{P}} \rho_{h}\left(\frac{\left\|z_{i}-z_{j}\right\|_{\epsilon}}{\left\|r_{\mathrm{c}}\right\|_{\epsilon}}\right)\left\|w_{j}-w_{i}\right\|^{2} .
\end{aligned}
$$

Substituting (27)-(29) into (26) yields, for all $(z, w) \in \mathcal{D}$,

$$
\dot{W}(z, w)=-\frac{1}{2} \sum_{(i, j) \in \mathcal{P}} \rho_{h}\left(\frac{\left\|z_{i}-z_{j}\right\|_{\epsilon}}{\left\|r_{\mathrm{c}}\right\|_{\epsilon}}\right)\left\|w_{j}-w_{i}\right\|^{2}-\frac{\gamma}{p_{\mathrm{f}}}\|w\|^{2},
$$

which is nonpositive.

To show (b), note that, for all $t \geq 0,(z(t), w(t)) \in \mathcal{D}$. Therefore, (23) and (30) imply that, for all $t \geq 0, W(z(t), w(t)) \geq 0$ and $\dot{W}(z(t), w(t)) \leq 0$. Thus, $\lim _{t \rightarrow \infty} W(z(t), w(t))$ exists. Since, for all $(i, j) \in \mathcal{P}, \psi\left(\left\|z_{j}-z_{i}\right\|_{\epsilon}\right) \leq \psi_{\mathrm{s}}(z)$, it follows from (23) that, for all $(z, w) \in \mathcal{D}$ and all $(i, j) \in \mathcal{P}, W(z, w) \geq \psi\left(\left\|z_{j}-z_{i}\right\|_{\epsilon}\right)+\|w\|^{2} / 2 \geq 0$. Since, for all $t \geq 0, W(z(t), w(t))$ is bounded, it follows that, for all $(i, j) \in \mathcal{P}$ and all $t \geq 0, z_{j}(t)-z_{i}(t)$ and $w(t)$ are bounded, which implies $q_{j}(t)-q_{i}(t)$ and $p_{i}(t)$ are bounded. Therefore, (8)-(10), (14), (15), and (18) imply that, for all $t \geq 0, g(q(t), p(t), z(t))$ is bounded. Note that, for all $(z, w) \in \mathcal{D}$,

$$
\begin{aligned}
\ddot{W}(z, w) \triangleq & \frac{\partial \dot{W}(z, w)}{\partial z} w-\frac{\partial \dot{W}(z, w)}{\partial w} g(q, p, z) \\
= & -\left.\frac{1}{4\left\|r_{\mathrm{c}}\right\|_{\epsilon}} \sum_{(i, j) \in \mathcal{P}} \frac{\mathrm{d} \rho_{h}(\eta)}{\mathrm{d} \eta}\right|_{\eta=\left\|z_{j}-z_{i}\right\|_{\epsilon} /\left\|r_{c}\right\|_{\epsilon}}\left\|w_{j}-w_{i}\right\|^{2} \sigma_{\epsilon}^{\mathrm{T}}\left(z_{i}-z_{j}\right)\left[w_{i}-w_{j}\right] \\
& +\sum_{(i, j) \in \mathcal{P}}\left[u_{j}\left(q, p, z_{j}\right)-u_{i}\left(q, p, z_{i}\right)\right]^{\mathrm{T}}\left[w_{j}-w_{i}\right]+\frac{2 \gamma}{p_{\mathrm{f}}} g^{\mathrm{T}}(q, p, z) w .
\end{aligned}
$$

Since $\mathrm{d} \rho_{h}(\eta) / \mathrm{d} \eta$ and $\sigma_{\epsilon}$ are bounded, and, for all $t \geq 0, g(q(t), p(t), z(t))$ and $w(t)$ are bounded, it follows that, for all $t \geq 0, \ddot{W}(z(t), w(t))$ is bounded, which implies $\dot{W}(z(t), w(t))$ is uniformly continuous. Since $\lim _{t \rightarrow \infty} W(z(t), w(t))$ exists and $\dot{W}(z(t), w(t))$ is uniformly 
continuous, Barbalat's lemma [26, Lemma 8.2] implies that $\lim _{t \rightarrow \infty} \dot{W}(z(t), w(t))=0$. Thus, (30) implies that $\lim _{t \rightarrow \infty} w(t)=0$, or equivalently, $\lim _{t \rightarrow \infty} p_{i}(t)=\chi$, which confirms (b).

To show (e), since $\phi$ and $\mathrm{d} \phi(\eta) / \mathrm{d} \eta$ are bounded; for all $t \geq 0,(z(t), w(t)) \in \mathcal{D}$; and, for all $(i, j) \in \mathcal{P}$ and all $t \geq 0, p_{i}(t), u_{i}\left(q(t), p(t), z_{i}(t)\right), w_{i}(t)$, and $q_{j}(t)-q_{i}(t)$ are bounded, it can be shown that, for all $t \geq 0$,

$$
\dot{u}_{i}\left(q(t), p(t), z_{i}(t)\right) \triangleq \frac{\partial u_{i}\left(q, p, z_{i}\right)}{\partial q} p+\frac{\partial u_{i}\left(q, p, z_{i}\right)}{\partial p} g(q, p, z)+\left.\frac{\partial u_{i}\left(q, p, z_{i}\right)}{\partial z_{i}} w_{i}\right|_{\left(q, p, z_{i}\right)=\left(q(t), p(t), z_{i}(t)\right)},
$$

is bounded. Therefore, $u_{i}\left(q(t), p(t), z_{i}(t)\right)$ is uniformly continuous. Since $\lim _{t \rightarrow \infty} p_{i}(t)$ exists and $\dot{p}_{i}(t)=u_{i}\left(q(t), p(t), z_{i}(t)\right)$ is uniformly continuous, [26, Lemma 8.2] implies that $\lim _{t \rightarrow \infty} u_{i}\left(q(t), p(t), z_{i}(t)\right)=0$, which confirms (e).

To show (g), note that, for all $(i, j) \in \mathcal{P},\left\|q_{j}-q_{i}\right\|_{\epsilon}=\left\|q_{i}-q_{j}\right\|_{\epsilon}$ and $\sigma_{\epsilon}\left(q_{j}-q_{i}\right)=-\sigma_{\epsilon}\left(q_{i}-q_{j}\right)$, and (8)-(10) imply that, for all $(q, p) \in \mathbb{R}^{n m} \times \mathbb{R}^{n m}, \sum_{i \in \mathcal{I}} A_{i}(q)+R_{i}(q)+C_{i}(q, p)=0$. Since, in addition, for all $t \geq 0,\left\|z_{i}(t)\right\|>r_{2}, \lim _{t \rightarrow \infty} u_{i}\left(q(t), p(t), z_{i}(t)\right)=0$, and $\lim _{t \rightarrow \infty} p_{i}(t)=\chi$, it follows from (14), (15), and (18) that

$0=\lim _{t \rightarrow \infty}\left(\sum_{i \in \mathcal{I}} u_{i}\left(q(t), p(t), z_{i}(t)\right)+\kappa\left(\left\|z_{i}(t)\right\|\right)\left[\chi-p_{i}(t)\right]\right)=\lim _{t \rightarrow \infty} \sum_{i \in \mathcal{I}}\left[\frac{\gamma}{\left\|z_{i}(t)\right\|} z_{i}(t)-\frac{\gamma}{p_{\mathrm{f}}} \chi\right]$,

which implies that $\lim _{t \rightarrow \infty} \sum_{i \in \mathcal{I}} z_{i}(t) /\left\|z_{i}(t)\right\|=n \chi / p_{\mathrm{f}}$. Since $\|\chi\|=p_{\mathrm{f}}$, it follows that $\lim _{t \rightarrow \infty} \sum_{i \in \mathcal{I}} z_{i}^{\mathrm{T}}(t) \chi /\left(\left\|z_{i}(t)\right\| p_{\mathrm{f}}\right)=n$. Assume, for contradiction, that there exists $k \in \mathcal{I}$ such that $\lim _{t \rightarrow \infty} z_{k}^{\mathrm{T}}(t) \chi /\left(\left\|z_{k}(t)\right\| p_{\mathrm{f}}\right) \neq 1$. Let $\delta_{1}>0$ be such that, for all $t \geq 0$, there exists $t_{1} \geq t$ such that $\left|z_{k}^{\mathrm{T}}\left(t_{1}\right) \chi /\left(\| z_{k}\left(t_{1}\right)|| p_{\mathrm{f}}\right)-1\right|>\delta_{1}$. Since $\lim _{t \rightarrow \infty} \sum_{i \in \mathcal{I}} z_{i}^{\mathrm{T}}(t) \chi /\left(\left\|z_{i}(t)\right\| p_{\mathrm{f}}\right)=n$, it follows that there exists $t_{2} \geq 0$ such that, for all $t \geq t_{2},\left|\sum_{i \in \mathcal{I}} z_{i}^{\mathrm{T}}(t) \chi /\left(|| z_{i}(t)|| p_{\mathrm{f}}\right)-n\right|<$ $\delta_{1}$. Since $\lim _{t \rightarrow \infty} z_{k}^{\mathrm{T}}(t) \chi /\left(\left\|z_{k}(t)\right\| p_{\mathrm{f}}\right) \neq 1$, it follows that there exists $t_{3} \geq t_{2}$ such that $\left|z_{k}^{\mathrm{T}}\left(t_{3}\right) \chi /\left(|| z_{k}\left(t_{3}\right)|| p_{\mathrm{f}}\right)-1\right|>\delta_{1}$, which implies that $z_{k}^{\mathrm{T}}\left(t_{3}\right) \chi /\left(\left\|z_{k}\left(t_{3}\right)\right\| p_{\mathrm{f}}\right)<1-\delta_{1}$ because $z_{k}^{\mathrm{T}} \chi /\left(\left\|z_{k}\right\| p_{\mathrm{f}}\right) \leq 1$. Since, for all $i \in \mathcal{I} \backslash\{k\}, z_{i}^{\mathrm{T}}\left(t_{3}\right) \chi /\left(\left\|z_{i}\left(t_{3}\right)\right\| p_{\mathrm{f}}\right) \leq 1$ and $z_{k}^{\mathrm{T}}\left(t_{3}\right) \chi /\left(\left\|z_{k}\left(t_{3}\right)\right\| p_{\mathrm{f}}\right)$ $<1-\delta_{1}$, it follows that

$$
\sum_{i \in \mathcal{I}} \frac{z_{i}^{\mathrm{T}}\left(t_{3}\right) \chi}{\left\|z_{i}\left(t_{3}\right)\right\| p_{\mathrm{f}}}=\frac{z_{k}^{\mathrm{T}}\left(t_{3}\right) \chi}{\left\|z_{k}\left(t_{3}\right)\right\| p_{\mathrm{f}}}+\sum_{i \in \mathcal{I} \backslash\{k\}} \frac{z_{i}^{\mathrm{T}}\left(t_{3}\right) \chi}{\left\|z_{i}\left(t_{3}\right)\right\| p_{\mathrm{f}}}<1-\delta_{1}+n-1=n-\delta_{1},
$$

or equivalently, $\left|\sum_{i \in \mathcal{I}} z_{i}^{\mathrm{T}}\left(t_{3}\right) \chi /\left(|| z_{i}\left(t_{3}\right)|| p_{\mathrm{f}}\right)-n\right|>\delta_{1}$, which is a contradiction. Thus, for all $i \in \mathcal{I}, \lim _{t \rightarrow \infty} z_{i}^{\mathrm{T}}(t) \chi /\left(\left\|z_{i}(t)\right\| p_{\mathrm{f}}\right)=1$, which implies that

$$
\lim _{t \rightarrow \infty}\left\|\frac{1}{\left\|z_{i}(t)\right\|} z_{i}(t)-\frac{1}{p_{\mathrm{f}}} \chi\right\|^{2}=2-2 \lim _{t \rightarrow \infty} \frac{z_{i}^{\mathrm{T}}(t) \chi}{\left\|z_{i}(t)\right\| p_{\mathrm{f}}}=0 .
$$

Therefore, $\lim _{t \rightarrow \infty} z_{i}(t) /\left\|z_{i}(t)\right\|=\chi / p_{\mathrm{f}}$, which confirms $(\mathrm{g})$.

To show (f), since, for all $t \geq 0,\left\|z_{i}(t)\right\|>r_{2}, \lim _{t \rightarrow \infty} p_{i}(t)=\chi$, and $\lim _{t \rightarrow \infty} z_{i}(t) /\left\|z_{i}(t)\right\|=$ $\chi / p_{\mathrm{f}}$, it follows from (10), (14), and (15) that $\lim _{t \rightarrow \infty} C_{i}(q(t), p(t))=0$ and $\lim _{t \rightarrow \infty}\left[\nu\left(\left\|z_{i}(t)\right\|\right)\right.$ $\left.\times z_{i}(t)+\kappa\left(\left\|z_{i}(t)\right\|\right) p_{i}(t)\right]=0$. Since, in addition, $\lim _{t \rightarrow \infty} u_{i}\left(q(t), p(t), z_{i}(t)\right)=0$, it follows from (18) that $0=\lim _{t \rightarrow \infty}\left[u_{i}\left(q(t), p(t), z_{i}(t)\right)-C_{i}(q(t), p(t))-\nu\left(\left\|z_{i}(t)\right\|\right) z_{i}(t)-\kappa\left(\left\|z_{i}(t)\right\|\right) p_{i}(t)\right]=$ $\lim _{t \rightarrow \infty}\left[A_{i}(q(t))+R_{i}(q(t))\right]$, which confirms (f).

To show (d), it follows from $(27)$ that $\partial \psi_{\mathrm{s}}(z) / \partial z_{i}=A_{i}^{\mathrm{T}}(q)+R_{i}^{\mathrm{T}}(q)$, which implies $\lim _{t \rightarrow \infty} \partial \psi_{\mathrm{s}}(z) /\left.\partial z_{i}\right|_{z=z(t)}=\lim _{t \rightarrow \infty}\left[A_{i}^{\mathrm{T}}(q(t))+R_{i}^{\mathrm{T}}(q(t))\right]=0$, which confirms (d).

To show (c), assume $r_{\mathrm{c}}$ is such that, for all $(i, j) \in \mathcal{P}$ and all $t \geq 0,\left\|r_{\mathrm{c}}\right\|_{\epsilon}>\| q_{j}(t)-$ 
$q_{i}(t) \|_{\epsilon} / h$. Define $f:(d, \infty) \rightarrow(0, \infty)$ by $f(\eta) \triangleq \phi\left(\|\eta\|_{\epsilon}-\|d\|_{\epsilon}\right) \sigma_{\epsilon}(\eta)=\phi\left(\|\eta\|_{\epsilon}-\|d\|_{\epsilon}\right) \eta /(1+$ $\left.\epsilon\|\eta\|_{\epsilon}\right)$. For all $\eta \in(d, \infty), \phi\left(\|\eta\|_{\epsilon}-\|d\|_{\epsilon}\right)>0$, which implies that, for all $\eta \in(d, \infty)$, $\mathrm{d} f(\eta) / \mathrm{d} \eta=(a+b) /\left(2\left(1+\left(\|\eta\|_{\epsilon}-\|d\|_{\epsilon}+c\right)^{2}\right)^{3 / 2}\right)\left(\eta /\left(1+\epsilon\|\eta\| \|_{\epsilon}\right)\right)^{2}+\phi\left(\|\eta\|_{\epsilon}-\|d\|_{\epsilon}\right) /\left(1+\epsilon\|\eta\|_{\epsilon}\right)^{3}>$ 0 .

Let $\delta>0$, and define $v_{\delta} \triangleq f(d(n-1)+\delta)$. Let $t_{1} \geq 0$ be such that, for all $t \geq t_{1}$, $\left\|A_{i}(q(t))+R_{i}(q(t))\right\| \leq v_{\delta} / n$, which exists because $\lim _{t \rightarrow \infty}\left[A_{i}(q(t))+R_{i}(q(t))\right]=0$. Let $t_{2} \geq t_{1}$, and it follows that

$$
\left\|A_{i}\left(q\left(t_{2}\right)\right)+R_{i}\left(q\left(t_{2}\right)\right)\right\| \leq \frac{v_{\delta}}{n} .
$$

For all $(i, j) \in \mathcal{P}$, define $Q(i, j) \triangleq q_{j}\left(t_{2}\right)-q_{i}\left(t_{2}\right)$. Assume, for contradiction, that there exists $(i, j) \in \mathcal{P}$ such that $\|Q(i, j)\|>d(n-1)+\delta$. Let $(\alpha, \beta) \in \mathcal{P}$ be such that $\|Q(\alpha, \beta)\|=\max _{(i, j) \in \mathcal{P}}\|Q(i, j)\|$. Since $\|Q(\alpha, \beta)\|>d(n-1)+\delta$ and, for all $\eta \in(d, \infty)$, $\mathrm{d} f(\eta) / \mathrm{d} \eta>0$, it follows that

$$
v_{\delta}=f(d(n-1)+\delta)<f(\|Q(\alpha, \beta)\|) .
$$

Let $l_{1}, l_{2}, \ldots, l_{n} \in \mathcal{I}$ be such that: (i) $l_{1}=\alpha$ and $l_{n}=\beta$; (ii) for all $(i, j) \in \mathcal{P}, l_{i} \neq l_{j}$; and (iii) for all $i \in \mathcal{I} \backslash\{n\}, Q^{\mathrm{T}}\left(l_{i}, l_{i+1}\right) Q(\alpha, \beta) \geq 0$. Since $\|Q(\alpha, \beta)\|>d(n-1)+\delta$, it follows from (iii) that

$$
\begin{aligned}
(d(n-1)+\delta)\|Q(\alpha, \beta)\|<\|Q(\alpha, \beta)\|^{2} & =\sum_{i=1}^{n-1} Q^{\mathrm{T}}\left(l_{i}, l_{i+1}\right) Q(\alpha, \beta) \\
& \leq(n-1) \max _{i \in \mathcal{I} \backslash\{n\}} Q^{\mathrm{T}}\left(l_{i}, l_{i+1}\right) Q(\alpha, \beta),
\end{aligned}
$$

which implies that there exists $\omega \in \mathcal{I} \backslash\{n\}$ such that

$$
\left(d+\frac{\delta}{n-1}\right)\|Q(\alpha, \beta)\|<Q^{\mathrm{T}}\left(l_{\omega}, l_{\omega+1}\right) Q(\alpha, \beta) .
$$

Define $\mathcal{I}_{1} \triangleq\left\{l_{1}, \ldots, l_{\omega}\right\}$ and $\mathcal{I}_{2} \triangleq\left\{l_{\omega+1}, \ldots, l_{n}\right\}$, which are nonempty. It follows from (iii) that, for all $(i, j) \in \mathcal{P}$ such that $i<j, Q^{\mathrm{T}}\left(l_{i}, l_{j}\right) Q(\alpha, \beta)=\sum_{k=i}^{j-1} Q^{\mathrm{T}}\left(l_{k}, l_{k+1}\right) Q(\alpha, \beta) \geq 0$. Thus, for all $i \in \mathcal{I}_{1}, Q^{\mathrm{T}}\left(i, l_{\omega}\right) Q(\alpha, \beta) \geq 0$, and, for all $j \in \mathcal{I}_{2}, Q^{\mathrm{T}}\left(l_{\omega+1}, j\right) Q(\alpha, \beta) \geq 0$. Therefore, for all $(i, j) \in \mathcal{I}_{1} \times \mathcal{I}_{2}, 0 \leq Q^{\mathrm{T}}\left(i, l_{\omega}\right) Q(\alpha, \beta)+Q^{\mathrm{T}}\left(l_{\omega+1}, j\right) Q(\alpha, \beta)=Q^{\mathrm{T}}(i, j) Q(\alpha, \beta)-$ $Q^{\mathrm{T}}\left(l_{\omega}, l_{\omega+1}\right) Q(\alpha, \beta)$, which implies that $Q^{\mathrm{T}}\left(l_{\omega}, l_{\omega+1}\right) Q(\alpha, \beta) \leq Q^{\mathrm{T}}(i, j) Q(\alpha, \beta)$. Thus, it follows from (33) that, for all $(i, j) \in \mathcal{I}_{1} \times \mathcal{I}_{2},(d+\delta /(n-1))\|Q(\alpha, \beta)\|<Q^{\mathrm{T}}(i, j) Q(\alpha, \beta) \leq$ $\|Q(i, j)\|\|Q(\alpha, \beta)\|$, which implies $Q^{\mathrm{T}}(i, j) Q(\alpha, \beta)>0$ and $\|Q(i, j)\|>d$. Therefore, $(4)$ and (6) imply that, for all $(i, j) \in \mathcal{I}_{1} \times \mathcal{I}_{2}, \phi\left(\|Q(i, j)\|_{\epsilon}-\|d\|_{\epsilon}\right)>0$ and $\sigma_{\epsilon}^{\mathrm{T}}(Q(i, j)) Q(\alpha, \beta)>0$, which implies that

$$
\Phi^{\mathrm{T}}(Q(i, j)) Q(\alpha, \beta)>0 .
$$

Since, for all $(i, j) \in \mathcal{P}$ and all $t \geq 0,\left\|r_{\mathrm{c}}\right\|_{\epsilon}>\left\|q_{j}(t)-q_{i}(t)\right\|_{\epsilon} / h$, or equivalently, $\left\|q_{j}(t)-q_{i}(t)\right\|\left\|_{\epsilon} /\right\| r_{\mathrm{c}} \|_{\epsilon}<h$, it follows from (5) that, for all $\left(z\left(t_{2}\right), w\left(t_{2}\right)\right) \in \mathcal{D}$ and all $(i, j) \in \mathcal{P}$, $\rho_{h}\left(\|Q(i, j)\|_{\epsilon} /\left\|r_{\mathrm{c}}\right\|_{\epsilon}\right)=1$. Since, in addition, for all $(i, j) \in \mathcal{P},\|Q(i, j)\|_{\epsilon}=\|Q(j, i)\|_{\epsilon}$ and $\sigma_{\epsilon}(Q(i, j))=-\sigma_{\epsilon}(Q(j, i))$, it follows from (8) and (9) that $\sum_{i \in \mathcal{I}_{1}} A_{i}\left(q\left(t_{2}\right)\right)+R_{i}\left(q\left(t_{2}\right)\right)=$ $\sum_{i \in \mathcal{I}_{1}} \sum_{j \in \mathcal{I} \backslash\{i\}} \Phi(Q(i, j))=\sum_{i \in \mathcal{I}_{1}} \sum_{j \in \mathcal{I}_{2}} \Phi(Q(i, j))$. Furthermore, since $\alpha=l_{1} \in \mathcal{I}_{1}$ and $\beta=l_{n} \in \mathcal{I}_{2}$, it follows from (31), (32), and (34) that

$$
v_{\delta}\|Q(\alpha, \beta)\|<f\left(\|Q(\alpha, \beta \|)\| Q(\alpha, \beta) \|=\Phi^{\mathrm{T}}(Q(\alpha, \beta)) Q(\alpha, \beta)\right.
$$




$$
\begin{aligned}
& \leq \sum_{i \in \mathcal{I}_{1}} \sum_{j \in \mathcal{I}_{2}} \Phi^{\mathrm{T}}(Q(i, j)) Q(\alpha, \beta) \\
& =\sum_{i \in \mathcal{I}_{1}}\left[A_{i}\left(q\left(t_{2}\right)\right)+R_{i}\left(q\left(t_{2}\right)\right)\right]^{\mathrm{T}} Q(\alpha, \beta) \\
& \leq \sum_{i \in \mathcal{I}_{1}}\left\|A_{i}\left(q\left(t_{2}\right)\right)+R_{i}\left(q\left(t_{2}\right)\right)\right\|\|Q(\alpha, \beta)\| \\
& \leq \sum_{i \in \mathcal{I}_{1}} \frac{v_{\delta}}{n}\|Q(\alpha, \beta)\| \\
& <v_{\delta}\|Q(\alpha, \beta)\|,
\end{aligned}
$$

which is a contradiction. Thus, for all $t \geq t_{1}, \max _{(i, j) \in \mathcal{P}}\left\|q_{j}(t)-q_{i}(t)\right\| \leq d(n-1)+\delta$, which confirms (c).

To show (a), assume $W(z(0), w(0))<\psi(0)$. Let $(i, j) \in \mathcal{P}$. Since, for all $t \geq 0$, $\psi\left(\left\|z_{j}(t)-z_{i}(t)\right\|_{\epsilon}\right) \leq \psi_{\mathrm{s}}(z(t)) \leq W(z(t), w(t))$, and $\dot{W}(z(t), w(t)) \leq 0$, it follows that, for all $t \geq 0, \psi\left(\left\|z_{j}(t)-z_{i}(t)\right\|_{\epsilon}\right) \leq W(z(t), w(t)) \leq W(z(0), w(0))<\psi(0)$. Therefore, for all $t \geq 0$, $\left\|z_{i}(t)-z_{j}(t)\right\|_{\epsilon} \neq 0$, which implies $q_{j}(t)-q_{i}(t) \neq 0$, which confirms (a).

\section{Appendix C. Proof of Lemma 1}

Proof of Lemma 1. Assume that, for all $(i, j) \in \mathcal{P},\left\|\xi_{j}-\xi_{i}\right\| \in\{d\} \cup\left[r_{\gamma}, \infty\right)$. Let $t_{0} \geq 0$, and assume $q\left(t_{0}\right)=\xi$ and $p\left(t_{0}\right)=0$. Since $q_{i}\left(t_{0}\right)=\xi_{i}$, it follows that, for all $(i, j) \in \mathcal{P},\left\|q_{j}\left(t_{0}\right)-q_{i}\left(t_{0}\right)\right\| \in\{d\} \cup\left[r_{\gamma}, \infty\right)$. We consider 2 cases: $r_{\alpha}=0$ and $r_{\alpha}>0$. First, assume $r_{\alpha}=0$, and it follows from (24) that $r_{\gamma}=r_{\mathrm{c}}$. Thus, for all $(i, j) \in \mathcal{P}$, $\left\|q_{j}\left(t_{0}\right)-q_{i}\left(t_{0}\right)\right\| \in\{d\} \cup\left[r_{\mathrm{c}}, \infty\right)$, which implies that $\mathcal{A}_{i}\left(t_{0}\right)=\left\{j \in \mathcal{I} \backslash\{i\}:\left\|q_{j}\left(t_{0}\right)-q_{i}\left(t_{0}\right)\right\|=d\right\}$. Therefore, (6) and (8) imply that $A_{i}\left(q\left(t_{0}\right)\right)=0$. Next, assume $r_{\alpha}>0$, and since $z_{i}\left(t_{0}\right)=0$, (M2) implies that $\mu\left(\left\|z_{i}\left(t_{0}\right)\right\|\right)=0$. Thus, for $r_{\alpha} \geq 0$, it follows that $\mu\left(\left\|z_{i}\left(t_{0}\right)\right\|\right) A_{i}\left(q\left(t_{0}\right)\right)=0$. Since, for all $(i, j) \in \mathcal{P},\left\|q_{j}\left(t_{0}\right)-q_{i}\left(t_{0}\right)\right\| \geq \min \left\{d, r_{\gamma}\right\}=d$, it follows that $\mathcal{R}_{i}\left(t_{0}\right)=\emptyset$, which implies from (9) that $R_{i}\left(q\left(t_{0}\right)\right)=0$. In addition, since $q\left(t_{0}\right)=\xi$ and $p\left(t_{0}\right)=0$, it follows that $C_{i}\left(q\left(t_{0}\right), p\left(t_{0}\right)\right)=0, \nu\left(\left\|z_{i}\left(t_{0}\right)\right\|\right) z_{i}\left(t_{0}\right)=0$, and $\kappa\left(\left\|z_{i}\left(t_{0}\right)\right\|\right) p_{i}\left(t_{0}\right)=0$. Therefore, $u\left(q\left(t_{0}\right), 0\right)=0$, which implies that $(\xi, 0)=\left(q\left(t_{0}\right), p\left(t_{0}\right)\right) \in \mathcal{E}$.

\section{Appendix D. Proof of Theorems 2 and 3}

Proof of Theorem 2. Let $i \in \mathcal{I}$, and assume that, for all $t \geq t_{0}$, (a) or (b) hold. Let $t_{1} \geq t_{0}$, and it follows that, for $t=t_{1}$, (a) or (b) hold.

First, assume that, for $t=t_{1}$, (a) holds, and it follows that $\mathcal{A}_{i}\left(t_{1}\right)=\mathcal{R}_{i}\left(t_{1}\right)=\mathcal{N}_{i}\left(t_{1}\right)=\emptyset$, which implies from (8)-(10) that $A_{i}\left(q\left(t_{1}\right)\right)=R_{i}\left(q\left(t_{1}\right)\right)=C_{i}\left(q\left(t_{1}\right), p\left(t_{1}\right)\right)=0$.

Next, assume that, for $t=t_{1}$, (b) holds and (a) does not hold. Thus, $\left\|z_{i}\left(t_{1}\right)\right\|<$ $\min \left\{r_{\alpha}, r_{\delta}\right\} \leq r_{\delta}$. Let $j \in \mathcal{N}_{i}\left(t_{1}\right)$, which exists because (a) does not hold. Since $\| q_{j}\left(t_{1}\right)-$ $q_{i}\left(t_{1}\right) \|<r_{\mathrm{c}}$, it follows from the assumption of Theorem 2 that $\left\|z_{j}\left(t_{1}\right)\right\|<\min \left\{r_{\alpha}, r_{\delta}\right\} \leq r_{\delta}$. Since $\left\|z_{i}\left(t_{1}\right)\right\|<r_{\delta}$ and $\left\|z_{j}\left(t_{1}\right)\right\|<r_{\delta}$, it follows that

$$
\begin{aligned}
d & =\min _{(k, l) \in \mathcal{P}}\left\|\xi_{l}-\xi_{k}\right\|-2 r_{\delta} \\
& \leq\left\|\xi_{j}-\xi_{i}\right\|-2 r_{\delta} \\
& =\left\|z_{j}\left(t_{1}\right)-z_{i}\left(t_{1}\right)+q_{j}\left(t_{1}\right)-q_{i}\left(t_{1}\right)\right\|-2 r_{\delta} \\
& \leq\left\|z_{j}\left(t_{1}\right)\right\|+\left\|z_{i}\left(t_{1}\right)\right\|+\left\|q_{j}\left(t_{1}\right)-q_{i}\left(t_{1}\right)\right\|-2 r_{\delta} \\
& <\left\|q_{j}\left(t_{1}\right)-q_{i}\left(t_{1}\right)\right\| .
\end{aligned}
$$


Therefore, $\mathcal{R}_{i}\left(t_{1}\right)=\emptyset$, which implies from $(9)$ that $R_{i}\left(q\left(t_{1}\right)\right)=0$. Since, in addition, $\left\|z_{i}\left(t_{1}\right)\right\|<\min \left\{r_{\alpha}, r_{\delta}\right\} \leq r_{\alpha}$, it follows from (M2) that $\mu\left(\left\|z_{i}\left(t_{1}\right)\right\|\right)=0$, which implies that $\mu\left(\left\|z_{i}\left(t_{1}\right)\right\|\right) A_{i}\left(q\left(t_{1}\right)\right)=\mu\left(\left\|z_{i}\left(t_{1}\right)\right\|\right) C_{i}\left(q\left(t_{1}\right), p\left(t_{1}\right)\right)=0$.

Thus, combining cases yields that

$$
\mu\left(\left\|z_{i}\left(t_{1}\right)\right\|\right) A_{i}\left(q\left(t_{1}\right)\right)+R_{i}\left(q\left(t_{1}\right)\right)+\mu\left(\left\|z_{i}\left(t_{1}\right)\right\|\right) C_{i}\left(q\left(t_{1}\right), p\left(t_{1}\right)\right)=0 .
$$

Therefore, for all $t \geq t_{0}, u_{i}\left(q(t), p(t), z_{i}(t)\right)=\nu\left(\| z_{i}(t)||\right) z_{i}(t)+\kappa\left(\left\|z_{i}(t)\right\|\right) p_{i}(t)$. Thus, part (b) of Proposition 2 implies that there exists $t_{2} \geq t_{0}$ such that, for all $t \geq t_{2}, \ddot{q}_{i}(t)+2 \zeta \omega_{\mathrm{n}} \dot{q}_{i}(t)+$ $\omega_{\mathrm{n}}^{2} q_{i}(t)=\omega_{\mathrm{n}}^{2} \xi_{i}$, which implies that $\lim _{t \rightarrow \infty} q_{i}(t)=\xi_{i}$ and $\lim _{t \rightarrow \infty} p_{i}(t)=0$.

Proof of Theorem 3. Since $r_{\alpha}=r_{\beta}=0$, it follows from (M1) that $\mu=1$, which implies that the derivative of $V(q, p)$ along the trajectories of (1), (2), and (18) is

$$
\begin{aligned}
& \dot{V}(q, p) \triangleq \frac{\partial V(q, p)}{\partial q} p \\
&=\sum_{i \in \mathcal{I}}\left(\frac{\partial \psi_{\mathrm{s}}(q)}{\partial q_{i}}+\frac{\partial}{\partial q_{i}}\left[\int_{0}^{\left\|\xi_{i}-q_{i}\right\|} \lambda \nu(\lambda) \mathrm{d} \lambda\right]+A_{i}^{\mathrm{T}}(q)\right. \\
&\left.\quad+R_{i}^{\mathrm{T}}(q)+C_{i}^{\mathrm{T}}(q, p)+\nu\left(\left\|z_{i}\right\|\right) z_{i}^{\mathrm{T}}+\kappa\left(\left\|z_{i}\right\|\right) p_{i}^{\mathrm{T}}\right) p_{i} .
\end{aligned}
$$

Since, for all $(k, j) \in \mathcal{P},\left\|q_{j}-q_{k}\right\|_{\epsilon}=\left\|q_{k}-q_{j}\right\|_{\epsilon}$, it follows that $\psi\left(\left\|q_{j}-q_{k}\right\|_{\epsilon}\right)=\psi\left(\left\|q_{k}-q_{j} \mid\right\|_{\epsilon}\right)$. Therefore, (4), (8), (9), (21), and (22) imply that, for all $i \in \mathcal{I}$,

$$
\begin{aligned}
\frac{\partial \psi_{\mathrm{s}}(q)}{\partial q_{i}}=\frac{\partial}{\partial q_{i}}\left[\sum_{k=1}^{n-1} \sum_{j=k+1}^{n} \psi\left(\left\|q_{j}-q_{k}\right\|_{\epsilon}\right)\right] & =\sum_{j \in \mathcal{I} \backslash\{i\}} \frac{\partial \psi\left(\left\|q_{j}-q_{i}\right\|_{\epsilon}\right)}{\partial q_{i}} \\
& =-\sum_{j \in \mathcal{I} \backslash\{i\}} \rho_{h}\left(\frac{\left\|q_{j}-q_{i}\right\|_{\epsilon}}{\left\|r_{c}\right\|_{\epsilon}}\right) \Phi^{\mathrm{T}}\left(q_{j}-q_{i}\right) \\
& =-A_{i}^{\mathrm{T}}(q)-R_{i}^{\mathrm{T}}(q) .
\end{aligned}
$$

Define $\nu^{\prime}(\eta) \triangleq \nu(\sqrt{\eta})$, and it follows that $\int_{0}^{\left\|\xi_{i}-q_{i}\right\|} \lambda \nu(\lambda) \mathrm{d} \lambda=\frac{1}{2} \int_{0}^{\left\|\xi_{i}-q_{i}\right\|^{2}} \nu^{\prime}(\tau) \mathrm{d} \tau$, where $\tau=\lambda^{2}$ and $\mathrm{d} \tau=2 \lambda \mathrm{d} \lambda$. Thus,

$$
\frac{\partial}{\partial q_{i}}\left[\int_{0}^{\left\|\xi_{i}-q_{i}\right\|} \lambda \nu(\lambda) \mathrm{d} \lambda\right]=\frac{\partial}{\partial q_{i}}\left[\frac{1}{2} \int_{0}^{\left\|\xi_{i}-q_{i}\right\|^{2}} \nu^{\prime}(\tau) \mathrm{d} \tau\right]=-\nu\left(\left\|\xi_{i}-q_{i}\right\|\right)\left[\xi_{i}-q_{i}\right]^{\mathrm{T}}=-\nu\left(\left\|z_{i}\right\|\right) z_{i}^{\mathrm{T}} .
$$

For all $(i, j) \in \mathcal{P}$ such that $\left\|q_{j}-q_{i}\right\|<r_{\mathrm{c}}$, it follows that $j \in \mathcal{N}_{i}$. Furthermore, it follows from (5) that, for all $(i, j) \in \mathcal{P}$ such that $\left\|q_{j}-q_{i}\right\| \geq r_{\mathrm{c}}, \rho_{h}\left(\left\|q_{j}-q_{i}\right\|_{\epsilon} /\left\|r_{\mathrm{c}}\right\|_{\epsilon}\right)=0$. Therefore, (10) implies that

$$
\begin{aligned}
\sum_{i \in \mathcal{I}} C_{i}^{\mathrm{T}}(q, p) p_{i} & =\frac{1}{2} \sum_{i \in \mathcal{I}} \sum_{j \in \mathcal{I} \backslash\{i\}} \rho_{h}\left(\frac{\left\|q_{j}-q_{i}\right\|_{\epsilon}}{\left\|r_{\mathrm{c}}\right\|_{\epsilon}}\right)\left[-p_{i}^{\mathrm{T}} p_{i}+p_{j}^{\mathrm{T}} p_{i}\right] \\
& =-\frac{1}{2} \sum_{(i, j) \in \mathcal{P}} \rho_{h}\left(\frac{\left\|q_{j}-q_{i}\right\|_{\epsilon}}{\left\|r_{\mathrm{c}}\right\|_{\epsilon}}\right)\left\|p_{j}-p_{i}\right\|^{2} .
\end{aligned}
$$

Substituting (36)-(38) into (35) yields $\dot{V}(q, p)=-\sum_{(i, j) \in \mathcal{P}} \rho_{h}\left(\left\|q_{j}-q_{i}\right\|_{\epsilon} /\left\|r_{\mathrm{c}}\right\|_{\epsilon}\right) \| p_{j}-$ $p_{i}\left\|^{2} / 2+\sum_{i \in \mathcal{I}} \kappa\left(\left\|z_{i}\right\|\right)\right\| p_{i} \|^{2}$, and since $\kappa<0$, it follows that $\dot{V}(q, p) \leq 0$.

To show (b), it can be shown that $\int_{0}^{\left\|\xi_{i}-q_{i}\right\|} \lambda \nu(\lambda) \mathrm{d} \lambda$ is radially unbounded in $q_{i}$, which implies that $V(q, p)$ is radially unbounded. Since, for all $(q, p) \in \mathbb{R}^{m} \times \mathbb{R}^{m}, \dot{V}(q, p) \leq 0$, it 
follows that, for all $t \geq 0, \dot{V}(q(t), p(t)) \leq 0$, which implies that $V(q(t), p(t))=V(q(0), p(0))+$ $\int_{0}^{t} \dot{V}(q(\tau), p(\tau)) \mathrm{d} \tau \leq V(q(0), p(0))$. Since $V(q, p)$ is positive semidefinite, radially unbounded, and, for all $t \geq 0, V(q(t), p(t)) \leq V(q(0), p(0))$, it follows that $q$ and $p$ are bounded.

Define $\Omega \triangleq\left\{(q, p) \in \mathbb{R}^{n m} \times \mathbb{R}^{n m}: V(q, p) \leq V(q(0), p(0))\right\}$, which is compact and positively invariant with respect to (1), (2), and (18). Furthermore, define $\Omega_{1} \triangleq\{(q, p) \in \Omega: \dot{V}(q, p)=$ $0\}=\{(q, p) \in \Omega: p=0\}$. Since $p=0$ implies that $\dot{p}=u(q, p)=0$, it follows that $\mathcal{M}$ is the largest set contained in $\Omega_{1}$ that is invariant with respect to (1), (2), and (18). Thus, LaSalle's invariance theorem $\left[26\right.$, Theorem 4.4] implies that, for all $q(0) \in \mathbb{R}^{n m}$ and $p(0) \in \mathbb{R}^{n m},(q, p)$ converges to $\mathcal{M}$, which confirms (b).

To show (a), assume that, for all $(i, j) \in \mathcal{P},\left\|\xi_{j}-\xi_{i}\right\|>r_{\mathrm{c}}$. Therefore, there exists $\delta>0$ such that, for all $(i, j) \in \mathcal{P},\left\|\xi_{j}-\xi_{i}\right\|>r_{\mathrm{c}}+\delta$. Define $\mathcal{D} \triangleq\left\{(q, p) \in \mathbb{R}^{n m} \times \mathbb{R}^{n m}:\|\xi-q\|<\delta / 2\right\}$, which implies that, for all $(q, p) \in \mathcal{D},\left\|z_{i}\right\| \leq\left(\sum_{i \in \mathcal{I}}\left\|z_{i}\right\|^{2}\right)^{1 / 2}=\|\xi-q\|<\delta / 2$. Thus, for all $(q, p) \in \mathcal{D}$ and all $(i, j) \in \mathcal{P}$

$$
r_{\mathrm{c}}<\left\|\xi_{j}-\xi_{i}||-\delta=\right\| z_{j}-z_{i}+q_{j}-q_{i}||-\delta \leq\left\|z_{j}\right\|+\left\|z_{i}\right\|+\left\|q_{j}-q_{i}||-\delta<\frac{\delta}{2}+\frac{\delta}{2}+\right\| q_{j}-q_{i}||-\delta=|| q_{j}-q_{i} \|,
$$

which implies $\left\|q_{j}-q_{i}\right\|_{\epsilon} /\left\|r_{\mathrm{c}}\right\|_{\epsilon}>1$. Hence, it follows from (5) and (21) that $\psi\left(\| q_{j}-\right.$ $\left.q_{i} \|_{\epsilon}\right)=\int_{\|d\|_{\epsilon}}^{\left\|r_{\epsilon}\right\|_{\epsilon}} \rho_{h}\left(s /\left\|r_{\mathrm{c}}\right\|_{\epsilon}\right) \phi\left(s-\|d\|_{\epsilon}\right) \mathrm{d} s$, which combined with $(22)$ implies that $\psi_{\mathrm{s}}(q)=$ $\frac{n(n-1)}{2} \int_{\|d\|_{\epsilon}}^{\left\|r_{\mathrm{c}}\right\|_{\epsilon}} \rho_{h}\left(s /\left\|r_{\mathrm{c}}\right\|_{\epsilon}\right) \phi\left(s-\|d\|_{\epsilon}\right) \mathrm{d} s$. Consider the candidate Lyapunov function $V_{1}: \mathcal{D} \rightarrow \mathbb{R}$ defined by $V_{1}(q, p) \triangleq V(q, p)-\psi_{\mathrm{s}}(q)$. It follows from $(25)$ that $V_{1}(\xi, 0)=0$. Since $\nu>0$, it follows that, for all $q_{i} \in \mathbb{R}^{m} \backslash\left\{\xi_{i}\right\}, \int_{0}^{\left\|\xi_{i}-q_{i}\right\|} \lambda \nu(\lambda) \mathrm{d} \lambda>0$. Therefore, (25) implies that, for all $(q, p) \in \mathcal{D} \backslash\{(\xi, 0)\}, V_{1}(q, p)>0$.

The derivative of $V_{1}(q, p)$ along the trajectories of $(1),(2)$, and (18) is $\dot{V}_{1}(q, p) \triangleq$ $\left[\partial V_{1}(q, p) / \partial q\right] p+\left[\partial V_{1}(q, p) / \partial p\right] u(q, p)=\dot{V}(q, p)$. Define $\mathcal{S} \triangleq\left\{(q, p) \in \mathcal{D}: \dot{V}_{1}(q, p)=0\right\}=$ $\{(q, p) \in \mathcal{D}: p=0\}$ and $E_{i} \triangleq\left[\begin{array}{lll}0_{m \times m(i-1)} & I_{m} & 0_{m \times m(n-i)}\end{array}\right] \in \mathbb{R}^{m \times n m}$, where $0_{l_{1} \times l_{2}}$ is the $l_{1} \times l_{2}$ matrix of zeros, and $I_{m}$ is the $m \times m$ identity matrix. Let $\tilde{q}:[0, \infty) \rightarrow \mathbb{R}^{n m}$ and $\tilde{p}:[0, \infty) \rightarrow \mathbb{R}^{n m}$ be such that, for all $t \geq 0,(\tilde{q}(t), \tilde{p}(t)) \in \mathcal{S}$ and, for all $i \in \mathcal{I}, \tilde{q}_{i}(t) \triangleq E_{i} \tilde{q}(t)$ and $\tilde{p}_{i}(t) \triangleq E_{i} \tilde{p}(t)$ satisfy (1), (2), and (18). Thus, $\tilde{p}(t) \equiv 0$. Since $\mathcal{S} \subset \mathcal{D}$, it follows that, for all $(i, j) \in \mathcal{P}$ and all $t \geq 0,\left\|\tilde{q}_{j}(t)-\tilde{q}_{i}(t)\right\|>r_{\mathrm{c}}$, which implies that $\rho_{h}\left(\left\|\tilde{q}_{j}(t)-\tilde{q}_{i}(t)\right\|\left\|_{\epsilon} /\right\| r_{\mathrm{c}} \|_{\epsilon}\right) \equiv$ 0 . Therefore, (8)-(10) imply that $A_{i}(\tilde{q}(t)) \equiv R_{i}(\tilde{q}(t)) \equiv C_{i}(\tilde{q}(t), \tilde{p}(t)) \equiv 0$. Since, in addition, $\tilde{p}(t) \equiv 0$, it follows from $(2)$ and $(18)$ that

$$
0 \equiv \dot{\tilde{p}}_{i}(t) \equiv u_{i}\left(\tilde{q}(t), \tilde{p}(t), \xi_{i}-\tilde{q}_{i}(t)\right) \equiv \nu\left(\left\|\xi_{i}-\tilde{q}_{i}(t)\right\|\right)\left[\xi_{i}-\tilde{q}_{i}(t)\right]
$$

which implies that $\tilde{q}_{i}(t) \equiv \xi_{i}$ because $\nu>0$. Thus, $(\tilde{q}(t), \tilde{p}(t)) \equiv(\xi, 0)$ is the only solution to (1), (2), and (18) such that, for all $t \geq 0,(\tilde{q}(t), \tilde{p}(t)) \in \mathcal{S}$. Therefore, [26, Corollary 4.1] implies that $(q(t), p(t)) \equiv(\xi, 0)$ is a locally asymptotically equilibrium of (1), (2), and (18), which confirms (a).

Finally, to show (c), assume $V(q(0), p(0))<\psi(0)$. Since, for all $(i, j) \in \mathcal{P}, \psi\left(\left\|q_{j}-q_{i}\right\|_{\epsilon}\right) \leq$ $\psi_{\mathrm{s}}(q) \leq V(q, p)$ and $\dot{V}(q, p) \leq 0$, it follows that, for all $t \geq 0, \psi\left(\left\|q_{j}(t)-q_{i}(t)\right\|_{\epsilon}\right) \leq$ $V(q(t), p(t)) \leq V(q(0), p(0))<\psi(0)$. Thus, for all $(i, j) \in \mathcal{P}$ and all $t \geq 0,\left\|q_{i}(t)-q_{j}(t)\right\|_{\epsilon} \neq 0$, which implies $q_{j}(t)-q_{i}(t) \neq 0$, which confirms (c).

[1] G. Punzo, P. Karagiannakis, D. J. Bennet, M. Macdonald, and S. Weiss. Enabling and exploiting self-similar central symmetry formations. IEEE Trans. Aerosp. Electron. Syst., 50(1):689-703, 2014. 
[2] D. M. Stipanović, G. Inalhan, R. Teo, and C. J. Tomlin. Decentralized overlapping control of a formation of unmanned aerial vehicles. Automatica, 40(8):1285-1296, 2004.

[3] R. M. Murray. Recent research in cooperative control of multivehicle systems. J. Dynam. Syst., Measure., Contr., 129(5):571-583, 2007.

[4] J. A. Fax and R. M. Murray. Information flow and cooperative control of vehicle formations. IEEE Trans. Autom. Contr., 49(9):1465-1476, 2004.

[5] G. Lafferriere, A. Williams, J. Caughman, and J. J. P. Veerman. Decentralized control of vehicle formations. Syst. Contr. Lett., 54(9):899-910, 2005.

[6] M. Guo, M. M. Zavlanos, and D. V. Dimarogonas. Controlling the relative agent motion in multi-agent formation stabilization. IEEE Trans. Autom. Contr., 59(3):820-826, 2014.

[7] Y. Cao and W. Ren. Distributed coordinated tracking with reduced interaction via a variable structure approach. IEEE Trans. Autom. Contr., 57(1):33-48, 2012.

[8] R. Vidal, O. Shakernia, and S. Sastry. Following the flock. IEEE Robot. Automat. Mag., 11(4):14-20, 2004.

[9] D. Gu and Z. Wang. Leader-follower flocking: Algorithms and experiments. IEEE Trans. Contr. Syst. Tech., 17(5):1211-1219, 2009.

[10] R. Olfati-Saber. Flocking for multi-agent dynamic systems: algorithms and theory. IEEE Trans. Autom. Contr., 51(3):401-420, 2006.

[11] H. Su, X. Wang, and Z. Lin. Flocking of multi-agents with a virtual leader. IEEE Trans. Autom. Contr., 54(2):293-307, 2009.

[12] H. Shi, L. Wang, and T. Chu. Flocking of multi-agent systems with a dynamic virtual leader. Int. J. Contr., 82(1):43-58, 2009.

[13] M. M. Zavlanos, M. B. Egerstedt, and G. J. Pappas. Graph-theoretic connectivity control of mobile robot networks. Proc. IEEE, 99(9):1525-1540, 2011.

[14] H. G. Tanner, A. Jadbabaie, and G. J. Pappas. Flocking in fixed and switching networks. IEEE Trans. Autom. Contr., 52(5):863-868, 2007.

[15] J. Park, H. J. Kim, and S. Ha. Cucker-Smale flocking with inter-particle bonding forces. IEEE Trans. Autom. Contr., 55(11):2617-2623, 2010.

[16] Y. Cao, W. Yu, W. Ren, and G. Chen. An overview of recent progress in the study of distributed multi-agent coordination. IEEE Trans. Ind. Informat., 9(1):427-438, 2013.

[17] W. Ren. On consensus algorithms for double-integrator dynamics. IEEE Trans. Autom. Contr., 53(6):1503-1509, 2008.

[18] W. Ren, R. W. Beard, and E. M. Atkins. Information consensus in multivehicle cooperative control. IEEE Contr. Syst., 27(2):71-82, 2007.

[19] W. Ni and D. Cheng. Leader-following consensus of multi-agent systems under fixed and switching topologies. Syst. Contr. Lett., 59(3-4):209-217, 2010.

[20] F. Cucker and S. Smale. Emergent behavior in flocks. IEEE Trans. Autom. Contr., 52(5):852-862, 2007.

[21] D. Panagou, D. M. Stipanović, and P. G. Voulgaris. Distributed coordination control for multi-robot networks using Lyapunov-like barrier functions. IEEE Trans. Autom. Contr., 61(3):617-632, 2016.

[22] D. V. Dimarogonas, M. M. Zavlanos, S. G. Loizou, and K . J. Kyriakopoulos. Decentralized motion control of multiple holonomic agents under input constraints. In Proc. Conf. Decision Contr., volume 4, pages 3390-3395, 2003.

[23] D. V. Dimarogonas, S. G. Loizou, K. J. Kyriakopoulos, and M. M. Zavlanos. A feedback stabilization and collision avoidance scheme for multiple independent non-point agents. Automatica, 42(2):229-243, 2006.

[24] H. G. Tanner. Flocking with obstacle avoidance in switching networks of interconnected vehicles. In Proc. IEEE Int. Conf. Robot. Automat., pages 3006-3011, 2004.

[25] C. W. Reynolds. Flocks, herds, and schools: A distributed behavioral model. Computer Graphics (SIGGRAPH '87 Conf. Proc.), 21(4):25-34, 1987.

[26] H. K. Khalil. Nonlinear Systems. Prentice Hall PTR, 2002. 